\title{
Perspektiven auf die Gestaltung von
}

\author{
Interaktionsarbeit
}

\section{Whitepaper}

Julia Friedrich, Kristin Gilbert, Ulrike Pietrzyk, Vanita Römer, Anne Steputat-Rätze, Christian Zinke-WehImann

Juli 2021 



\section{Inhalt}

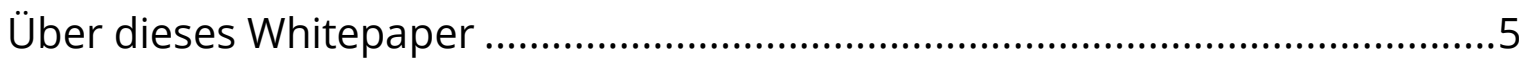

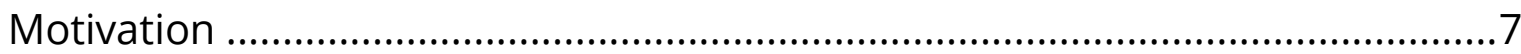

Herausforderungen bei der Gestaltung von Interaktionsarbeit................. 9

Arbeits- und dienstleistungswissenschaftliche Sicht auf Interaktionsarbeit

Gute Dienstleistungen bei guter Arbeit ...................................................14

Stand der Forschung von Arbeitswissenschaft und Dienstleistungs-

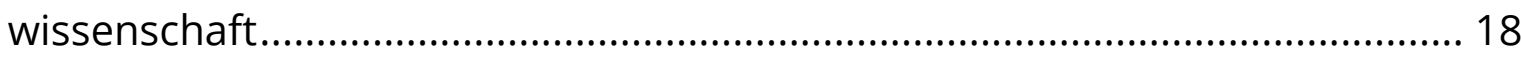

Selbstverständnis von Arbeitswissenschaft und

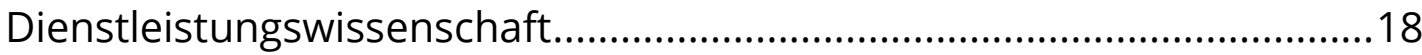

Interaktionsarbeit als Aspekt von Dienstleistungen.................................22

Besonderheiten der Dienstleistungen Pflege und Kinderbetreuung .......27

Technik und Digitalisierung bei der Gestaltung von Dienstleistungen ....30

Förderlandschaft Dienstleistungsgestaltung .................................................... 33

Methoden zur Gestaltung von Interaktionsarbeit............................................. 37

Expert:innenworkshop „Systematisch Human - Die Zukunft der

Gestaltung von Interaktionsarbeit" ...........................................................39

Methodenzusammenstellung für Interaktionsarbeit..................................42

Prinzipien des Social Service Engineering ........................................................ 56

Ausblick für die Gestaltung von Interaktionsarbeit............................................ 61

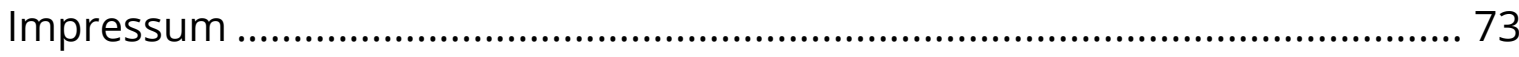

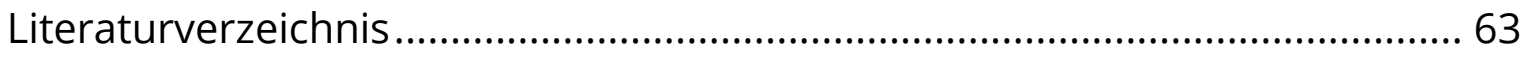





\section{Über dieses Whitepaper}

Ziel des Whitepapers ist es, die Notwendigkeit und die Grundlagen einer systematischen Verknüpfung von Arbeitswissenschaften und Dienstleistungswissenschaften im Sinne der Gestaltung nachhaltig humaner Arbeit in Zeiten der Digitalisierung darzustellen.

Zu diesem Zweck schaffen Wissenschaftspartner:innen der Arbeitsgruppe Wissen-Denken-Handeln der TU Dresden (Fakultät Psychologie) und der Abteilung Forschung und Entwicklung des Universitätsrechenzentrums der Universität Leipzig gemeinsam innerhalb des Projektes SO-SERVE (vom BMBF gefördert/ESF-kofinanziert) einen neuen Ansatz für die Gestaltung von Interaktionsarbeit: Das Social Service Engineering. Es integriert arbeitswissenschaftliche Gestaltungslösungen mit dem effizienzorientierten Ansatz des Service Engineering. Die Prinzipien des wissenschaftlich fundierten, praktisch erprobten und übertragbaren Social Service Engineering bilden die Grundlage für eine menschengerechte und wirtschaftliche Gestaltung von Interaktionsarbeit.

Das Projekt SO-SERVE lebt von der Mitarbeit unserer Praxispartner:innen: die Bosold Pflege GmbH, die KINDERVEREINIGUNG LEIPZIG e. V. und die vital.services $\mathrm{GmbH}$. An dieser Stelle möchten wir diesen für ihr außergewöhnliches Engagement, besonders unter den Einschränkungen durch die Corona-Pandemie, unseren herzlichen Dank aussprechen. 
Ebenso gilt es, den interdisziplinären Teilnehmenden am Expert:innenworkshop "Systematisch Human - Die Zukunft der Gestaltung von Interaktionsarbeit" für den konstruktiven interdisziplinären Austausch und den wertvollen Input zu danken. Die Erkenntnisse dieses Austauschs flossen in das vorliegende Whitepaper ein. Wir bedanken uns insbesondere bei:

\begin{tabular}{|c|c|}
\hline Name & Organisation \\
\hline Bartelheimer, Christian & Universität Paderborn \\
\hline Bartetzko, Marit & ATB Arbeit, Technik und Bildung gGmbH \\
\hline Dr. Ernst, Gerhard & $\begin{array}{l}\text { Humane Gestaltung von Arbeit und } \\
\text { Leben e. V. }\end{array}$ \\
\hline Dr. Freitag, Mike & $\begin{array}{l}\text { Fraunhofer-Institut für Arbeitswirtschaft } \\
\text { und Organisation }\end{array}$ \\
\hline Guerrero, Ricardo & Jacobs University Bremen \\
\hline Prof. Dr. Kieffer-Radwan, Sonja & Fachhochschule des Mittelstands \\
\hline Koch, Isabell & $\begin{array}{l}\text { Diakonisches Werk im Kirchenkreis Halber- } \\
\text { stadt e. V. }\end{array}$ \\
\hline Mewes, Eric & Otto-von-Guericke Universität \\
\hline Dr. Meyer, Kyrill & $\begin{array}{l}\text { IFDT - Institut für Digitale Technologien } \\
\text { gGmbH }\end{array}$ \\
\hline Middendorf, Hanna & Technische Universität Dortmund \\
\hline Müller, Jennifer & Universität Paderborn \\
\hline Dr. Peters, Christoph & Universität Kassel \\
\hline Pfahl, Sebastian & METOP GmbH \\
\hline Raeisy, Laleh & Technische Universität Dresden \\
\hline $\begin{array}{l}\text { Prof. Dr. Robra-Bissantz, } \\
\text { Susanne }\end{array}$ & Technische Universität Braunschweig \\
\hline Prof. Dr. Stollenwerk, Nicole & Fachhochschule des Mittelstands \\
\hline Sanders, Tobias & ATB Arbeit, Technik und Bildung gGmbH \\
\hline Sauter, Christine & $\begin{array}{l}\text { IFDT - Institut für Digitale Technologien } \\
\text { gGmbH }\end{array}$ \\
\hline Prof. Uhlmann, Michael & ATB Arbeit, Technik und Bildung gGmbH \\
\hline Wünnemann, Louisa & $\begin{array}{l}\text { Bundesanstalt für Arbeitsschutz und Ar- } \\
\text { beitsmedizin }\end{array}$ \\
\hline
\end{tabular}




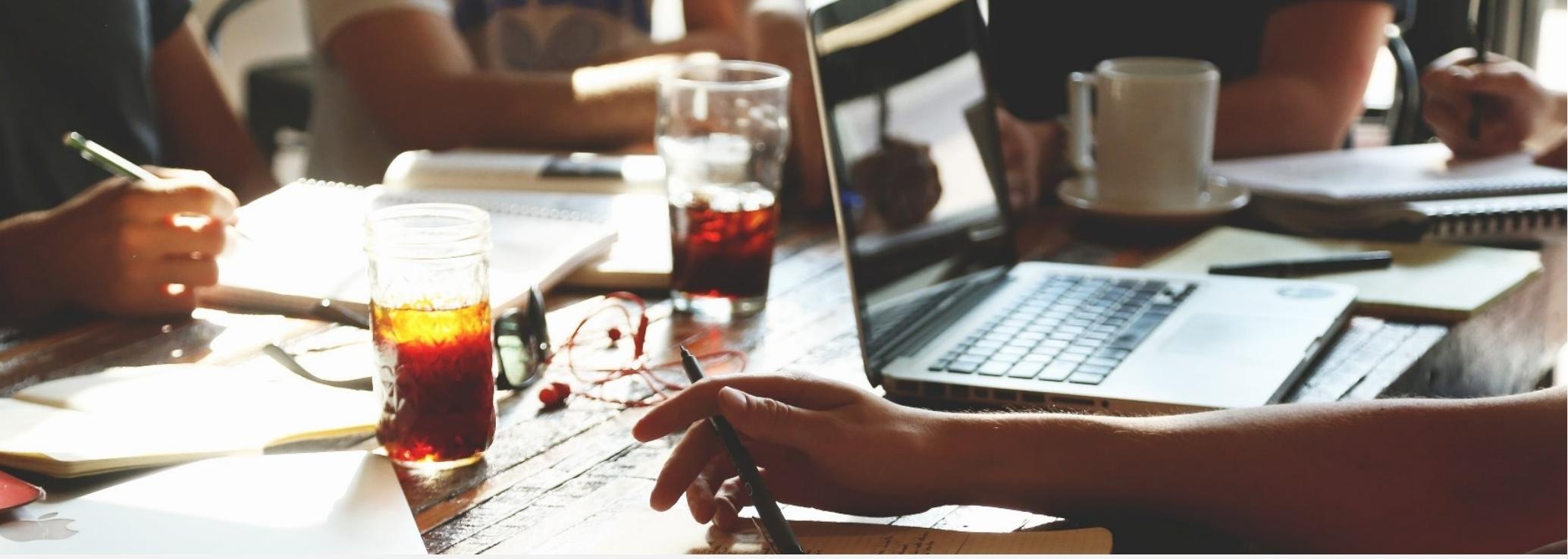

\section{Motivation}

In Deutschland arbeiten 63 Prozent der Beschäftigten sehr häufig oder oft in direktem Kontakt mit Menschen (vgl. Institut DGB-Index Gute Arbeit, 2018). Diese als Interaktionsarbeit bezeichnete Arbeit an und mit Menschen findet nicht ausschließlich in sozialen und personenbezogenen Dienstleistungen (DL) statt (bspw. in der Pflege oder Sozialarbeit), sondern überall dort, wo Menschen in der Rolle eines DL-Nehmenden mit Anbieter:innen eines Produktes oder einer DL in Kontakt treten (bspw. im Gastgewerbe oder im Handel).

Der Kern von Interaktionsarbeit besteht in der Interaktion zwischen DL-Geber:innen (z. B. Verkäufer:innen) und DL-Empfänger:innen (z. B. Kund:innen) (Abbildung 1).

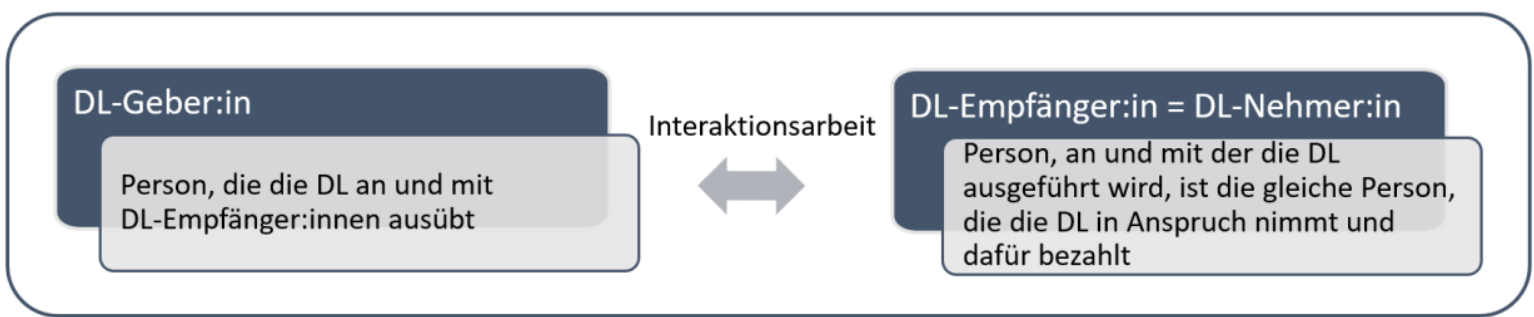

Abbildung 1: Interaktionsdyade

Bei einigen DL - wenn die DL-Empfänger:innen (z. B. Kita-Kinder) nicht mit den DL-Nehmer:innen Person (z. B. Eltern) zusammenfallen - tritt auch die Interaktion zwischen DL-Geber:innen (z. B. Erzieher:innen) und DL-Nehmer:innen in den Fokus der Gestaltung von Interaktionsarbeit (Abbildung 2). 


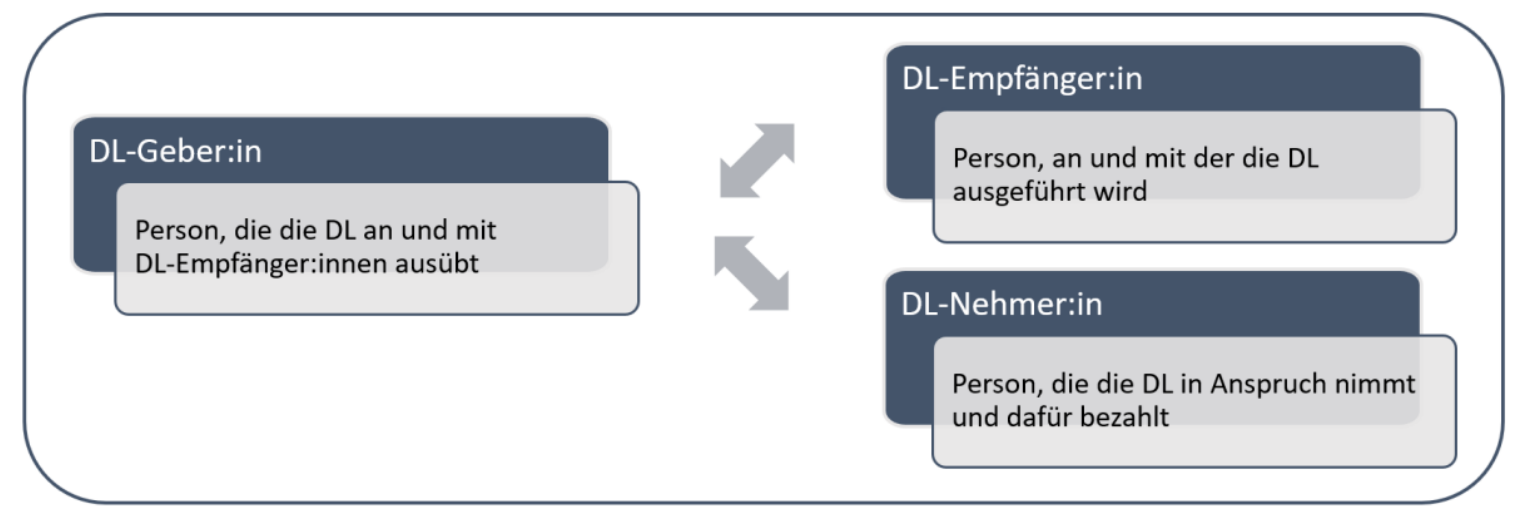

Abbildung 2: Interaktionstriade

Interaktionsarbeit ist nicht einfach eine Integration von Ressourcen wie in der Arbeit am Gegenstand, sondern muss darüber hinaus die Ziele, Wünsche, Meinungen und momentane (emotionale, physische) Verfassungen der Beteiligten berücksichtigen. Interaktionsarbeit ist damit ein Arbeiten mit und an Menschen, welches für alle Menschen Wert erzeugt und gleichzeitig Qualitätsansprüchen gerecht werden soll. Für eine gelungene Interaktionsarbeit ist dementsprechend eine erfolgreiche Abstimmung der Interagierenden im Sinne einer Koproduktion (Value-Co-Creation) erforderlich. Die menschengerechte Interaktion erfordert daher individuelle Handlungsspielräume, Spontanität und Kreativität. Das Einräumen von Tätigkeitsspielräumen hat sich bei Interaktionsarbeit als eine unerlässliche Gestaltungsnotwendigkeit erwiesen (vgl. Hacker, 2009).

Insbesondere in personenbezogenen $\mathrm{DL}$ geht die Interaktionsarbeit mit Arbeitsanforderungen einher, die die Arbeitsintensität, die Arbeitszeit (z. B. Überstunden, atypische Arbeitszeiten) sowie emotionale Anforderungen (z. B. eigene Gefühle zu verbergen) betreffen (vgl. Müller \& Thorein, 2017). Neben dem Bedarf an nachhaltigen und qualitativ hochwertigen DL-Systemen besteht bei Interaktionsarbeit ein hoher Ökonomisierungsdruck. Bestrebungen der Qualitätssicherung gehen oft mit zunehmenden Dokumentationspflichten einher, welche die Belastungssituation der Beschäftigten ebenso verschärfen wie Budgetkürzungen. Aus diesen Bedingungen ergeben sich für die Unternehmen Gestaltungsbedarfe, die sich sowohl an den Kriterien menschengerechter Arbeit als auch an Effizienzkriterien orientieren müssen. 


\section{Herausforderungen bei der Gestaltung von Interaktionsarbeit}

Für die menschengerechte Arbeitsgestaltung existieren wissenschaftlich fundierte Grundsätze, die Eingang in Normen gefunden haben (z. B. DIN EN ISO 6385:2016-12, DIN EN ISO 10075-2:2000). Die beispielhaft genannten Normen gelten für unterschiedliche Arten menschlicher Arbeitstätigkeiten, und damit auch für die Interaktionsarbeit. Auch Hacker (2018a, S. 35) weist darauf hin: "Wissenschaftlich gesicherte Ansatzpunkte und Ziele einer menschengerechten Arbeitsgestaltung sind auch für Interaktionsarbeit bis hin zu verbindlichen internationalen Standards bekannt". Ebenso gibt es Normierungsansätze, welche Qualitätskriterien etwa für soziale DL definieren, z. B. die DIN 77800 "Qualitätsanforderungen an Anbieter der Wohnform „Betreutes Wohnen für ältere Menschen“, die DIN EN 15224 „Dienstleistungen in der Gesundheitsversorgung - Qualitätsmanagementsysteme“ sowie Kriterien für das Qualitätsmanagement im Hinblick auf die Kund:innenzufriedenheit in DL (DIN ISO 10001 10004).

Bei der Realisierung der in den Normen genannten Grundsätze besteht eine große Herausforderung in der konkreten Auswahl und Umsetzung von Methoden und Gestaltungsmaßnahmen. Die Individualität der Interaktionen sowie die Subjektrolle der DL-Empfänger:innen bedingen, dass erprobte Analyse- und Gestaltungskonzepte aus der objekt- bzw. produktbezogenen Arbeit nicht uneingeschränkt auf die Gestaltung von Interaktionsarbeit übertragen werden können. Beispielsweise sind die im Arbeits- und Gesundheitsschutz gewählten Vorgehensweisen des "Beseitigens" und des „Abschirmens vor Gefährdungen“ zwar für den Umgang mit Gefahrstoffen umsetzbar, allerdings kaum bei emotional beanspruchenden Situationen im Umgang mit DL-Empfänger:innen. Daher erhalten bei Interaktionsarbeit verhaltensbezogene Maßnahmen, wie das Erlernen individueller Bewältigungsstrategien (z. B. Detachment-Strategien, Schulung zum Umgang mit schwierigen Kund:innen), eine größere Bedeutung als in der produktorientierten Arbeit. Eine gezielte und passgenaue Verhaltensprävention, die über die gängigen Motivationsseminare hinausgeht, ist für Interaktionsarbeit daher unverzichtbar (Hacker, 2018b). 
Trotz der Forderungen nach einer guten Verhaltensprävention ist bei der Gestaltung von Interaktionsarbeit die Grundregel „Verhältnisprävention vor Verhaltensprävention“ (vgl. §4 Arbeitsschutzgesetz) einzuhalten. Die verhaltensbezogenen Maßnahmen zielen auf einen verbesserten Umgang mit den gegebenen Arbeitsanforderungen ab, nicht jedoch auf eine Verbesserung der Arbeitsbedingungen an sich. Die verhältnisbezogenen Maßnahmen können bspw. die Arbeitsteilung zwischen Menschen, die Funktionsteilung zwischen Mensch und Technik sowie arbeitsorganisatorische Maßnahmen umfassen. Derzeit besteht jedoch ein Mangel an ausreichend erprobten verhältnisbezogenen Arbeitsgestaltungsmaßnahmen für die Gestaltung von Interaktionsarbeit, die an nützliche verhaltensbezogene Maßnahmen gekoppelt sind (vgl. Hacker, 2018b).

Auch die für die Produktion entwickelten effizienzorientierten Methoden des Service Engineering, insbesondere der Prozessmodellierung und Standardisierung (Modularisierung und Produktisierung ${ }^{1}$ von DL) sind im Kontext der Interaktionsarbeit bzw. der personenbezogenen DL nur bedingt einsetzbar: Auf der einen Seite ermöglichen sie Routine, Wiederholbarkeit und Effizienzsteigerung. Auf der anderen Seite kann eine zu starke Fokussierung auf Standardisierung im Kontext der Interaktionsarbeit jedoch zu Unzufriedenheit sowohl auf Seiten der DL-Geber:innen als auch auf Seiten der DL-Empfänger:innen führen, weil Handlungsspielräume und flexibles Eingehen auf die sich zum Teil schnell ändernden Bedürfnisse der DL-Empfänger:innen (Individualisierung) eingeschränkt werden. Daher muss die Formalisierung der Arbeit und Standarisierung im Kontext der Interaktionsarbeit wohlüberlegt umgesetzt werden. Es gilt zu beachten, dass ein wichtiges Maß für die Qualität der erbrachten DL das Eingehen auf die Bedürfnisse der DL-Empfänger:innen ist. Diese DL-Qualität ist abhängig von der Möglichkeit einer flexiblen Umsetzung der DL und den Fähigkeiten der DL-Geber:innen, diesen Spielraum zu nutzen.

Im Service Engineering konzentrieren sich viele Arbeiten darauf, das DL-System zu gestalten, um größtmöglichen (ökonomischen) Nutzen zu erzeugen. Daraus

\footnotetext{
${ }^{1}$ Produktisierung von DL bezeichnet die Vorgehensweise DL durch Standardisierung in eine produktähnliche Form zu überführen, welche durch eine beständige Qualität, geringe Varianz, einfache Vervielfältigung und Vorhersehbarkeit von Kosten gekennzeichnet ist (vgl. Chattopadhyay 2012).
} 
resultiert eine Lücke in der Perspektive des Service Engineering für die Gestaltung von Interaktionsarbeit. Diese manifestiert sich in der mangelhaften Darstellung der Perspektive der Beschäftigten (wie sie bspw. in den Arbeitswissenschaften abgebildet wird) und der wenig detaillierten Betrachtung der Interaktion. Gestaltung von Interaktionsarbeit im Service Engineering bleibt derzeit häufig beschränkt auf die Rahmenbedingungen der Interaktion. So werden beispielsweise Kund:innen-Kontaktpunkte analysiert und gestaltet (i. S. eines Touchpoint Management) und die Kund:innen als Zielgruppe untersucht und dargestellt (z. B. Erstellung von Personas). Auf diese Weise können zielgruppenorientierte $\mathrm{DL}$ entwickelt und verbesserte Kund:innen-Erlebnisse geschaffen werden. Die Gestaltung der zwischen DL-Geber:innen und DL-Empfänger:innen stattfindenden Interaktion und vor allem die Mechanismen zur Realisierung guter Arbeit stehen im Service Engineering allerdings im Hintergrund. 


\section{Arbeits- und dienstleistungswissenschaftliche Sicht auf Interaktionsarbeit}

Arbeitswissenschaftliche Konzepte wie das Konzept der "dialogisch-interaktiven Erwerbsarbeit" (Hacker, 2009), der "interaktiven Arbeit" (Dunkel \& Weihrich, 2012) oder das integrative "Konzept der Interaktionsarbeit" (Böhle \& Weihrich, 2020) beschreiben Arbeitsmerkmale und Arbeitsanforderungen an Beschäftigte, die Interaktionsarbeit ausüben. Die Arbeitswissenschaft nimmt daher in der Betrachtung von DL die Perspektive der DL-Geber:innen ein und zielt darauf ab, für Beschäftigte "optimale Arbeitsbedingungen im Hinblick auf Gesundheit und Sicherheit, Wohlbefinden, Leistung und Effektivität zu schaffen, sowie Über- wie Unterforderung vorzubeugen“ (vgl. DIN EN ISO 10075-2:2000, S. 2). Die Perspektive der DL-Empfänger:innen, der DL-Nehmer:innen und Rationalisierungsziele spielen in der Arbeitswissenschaft gegenwärtig eine eher nachgeordnete Rolle, auch wenn die Arbeitswissenschaft in ihren Anfängen von den Lehren F. W. Taylors ausgehend - auf Rationalisierung ausgerichtet war (vgl. Schultetus, 2006). Es besteht heute die Auffassung, dass menschengerecht gestaltete Arbeit die Effizienz des gesamten DL-Systems verbessert (vgl. DIN EN ISO 6385:2016-12).

Im Gegensatz zur an der menschengerechten Arbeitsgestaltung orientierten Sichtweise der Arbeitswissenschaft zielt das Service Engineering auf die Gestaltung und Optimierung von DL-Prozessen ab und legt dabei den Fokus auf die DL-Empfänger:innen-Perspektive. DL-Empfänger:innen und DL-Nehmer:innen als Koproduzent:innen der DL werden als „Faktor Mensch“ betrachtet und gemeinsam mit den eigentlichen Informationsartefakten (Prozesse, Dokumente, Modelle) nicht aus der Perspektive der Arbeitsgestaltung (im Sinne "guter Arbeit"), sondern im Hinblick auf ihr Effizienzpotential analysiert (im Sinne "guter Dienstleistungen“). Zentrale Aspekte der Interaktionsarbeit wie Emotionen, zwischenmenschliche Beziehungen oder der Umgang mit Unwägbarkeiten, sind in dem Sinne der "blinde Fleck" des Service Engineering (vgl. Haab et al., 2019).

Bislang stehen die beiden Perspektiven zur Betrachtung von Interaktion, die Sicht der Arbeitsgestaltung und der Blickwinkel der DL-Optimierung, weitestgehend unverbunden nebeneinander. Derzeit fehlt für die menschengerechte 
und effiziente Gestaltung von Interaktionsarbeit ein "gemeinsamer Standard", der erfolgreiche Vorgehensweisen definiert (vgl. Ernst \& Zühlke-Robinet, 2018). Obwohl sich der Gestaltungsgegenstand („Interaktionsarbeit“) und die Fragestellungen der beiden Disziplinen ähneln und überschneiden, greifen Vertreter:innen der Disziplinen primär auf ihre eigenen Ansätze und Methoden zurück. 


\section{Gute Dienstleistungen bei guter Arbeit}

Eine Integration von Arbeitswissenschaft und Service Engineering erfordert nicht nur eine Verknüpfung von methodischen Vorgehensweisen aus den Disziplinen, sondern zudem eine Zusammenführung der primären Gestaltungsziele.

Während die Arbeitswissenschaft eine humane Arbeitsgestaltung für die DLGeber:innen anstrebt, zielt die DL-Wissenschaft auf eine hohe Qualität und Produktivität der $\mathrm{DL}$, die zu einer hohen Zufriedenheit der DL-Empfänger:innen/DL-Nehmer:innen führen sollen.

Humane resp. menschengerechte Arbeitsgestaltung bedeutet, dass die sogenannten Humankriterien der Arbeit eingehalten werden (vgl. DIN EN ISO 9241219, 2019, DIN EN ISO 614-2: 2008, DIN EN ISO 6385: 2016):

- Vermeidung von Über- und Unterforderung: Einer quantitativen (arbeitsmengenbezogene) und qualitativen (qualifikationsbezogenen) Über- oder Unterforderung bei der Interaktionsarbeit ist vorzubeugen (z. B. durch Auswahl benutzungsorientierter Software, Qualifizierung).

- Anforderungsvielfalt: Die Arbeitsaufgabe soll aus abwechslungsreichen Teilaufgaben bestehen, die möglichst viele verschiedene (motorische, intellektuelle, soziale) Kenntnisse und Fertigkeiten beanspruchen. Repetitive, einseitige Tätigkeiten sollen vermieden werden (z. B. durch Job Rotation, Job Enlargement).

- Handlungsspielraum: Es soll ein angemessener Grad an Entscheidungsfreiheit hinsichtlich Reihenfolge, Vorgehensweise und Arbeitstempo bei der Erledigung der Arbeitsaufgaben gewährt werden (z. B. durch Einräumen von Wahlmöglichkeiten bei der Tätigkeitsausführung).

- Ganzheitlichkeit: Die durchgeführten Arbeitsaufgaben sollen für die DL-Geber:innen als ganze Arbeitseinheiten und nicht nur als Teilstücke erkennbar sein. Neben der Ausführung sollen zur Arbeitsaufgabe auch vorbereitende und das Ergebnis kontrollierende Arbeitsaufgaben gehören (z. B. durch Reorganisation von Arbeitsprozessen). 
- Bedeutsamkeit: Die durchgeführten Arbeitsaufgaben sollen einen wesentlichen, für DL-Geber:innen verständlichen Beitrag zum gesamten Arbeitssystem aufweisen. DL-Geber:innen können die Bedeutung ihrer Arbeit erkennen, indem sie sehen, inwiefern ihre Tätigkeiten Anderen nützen, mit den Tätigkeiten Anderer zusammenhängen und zu den Zielen des Unternehmens beitragen.

- Rückmeldung: DL-Geber:innen sollen ausreichende, aussagekräftige Rückmeldungen über die Aufgabenerfüllung bzw. ihre Arbeitsleistung erhalten. Die Rückmeldungen können von anderen Personen (z. B. Führungskräften) stammen oder aus dem Arbeitsprozess selbst hervorgehen (z. B. über die Wahrnehmung von Arbeitsergebnissen).

- Lern- und Entwicklungsmöglichkeiten: Die Arbeitsaufgaben sind so gestaltet, dass bestehende Qualifikationen der DL-Geber:innen erhalten bleiben (Qualifikationsnutzung) und erweitert werden können (z. B durch herausfordernde Arbeitsaufgaben oder Qualifizierung).

Hohe Qualität und Produktivität im Sinne "guter Dienstleistung" können auf verschiedenen Ebenen hergestellt werden:

- Einnehmen der DL-Empfänger:innen-Perspektive: Mittels kundschaftsorientierter Methoden (z. B. Empathy Map, Customer Journey) der systematischen DL-Entwicklung können DL mit einer gleichbleibend hohen Qualität und Effizienz erbracht werden.

- Systematische Entwicklung: Aufgabe des Service Engineering ist die Entwicklung von Methoden, welche Anbieter:innen dabei unterstützen, ihre DL mit einer gleichbleibend hohen Qualität zu erbringen. Dies geschieht etwa über das Definieren fester Qualitätsmerkmale oder das Festlegen effizienter Abläufe der DL-Erbringung,

- Qualitätsmanagement: Die Qualität wird durch die systematische Gestaltung der Leistung kontrollierbar, hierbei kann unterschieden (Bruhn, 2020) werden zwischen: 
- Herstellung von Potentialqualität (bspw. durch nutzerfreundliche Technik, ansprechendes Ambiente der räumlichen Gestaltung, Sicherstellung der fachlichen, sozialen und kommunikativen Kompetenzen)

- Herstellung von Prozessqualität (bspw. durch gut aufeinander abgestimmte Prozesse, Maßnahmen zur Vermeidung von Doppelarbeit oder zur Fehlerreduktion)

- Herstellung von Ergebnisqualität (bspw. durch Individualisierung, Vollständigkeit, Termineinhaltung, Kulanzangebote)

- Produktisierung und Individualisierung (Jaakkola, 2011): Die Entwicklung von DL bewegt sich stets im Spannungsfeld zwischen der Produktisierung (bzw. Standardisierung) und der Individualisierung von DL-Prozessen und Angeboten. Das heißt, dass die Gestaltung einer "guten DL" sowohl die Wünsche / Ziele der DL-Anbieter:innen als auch der DL-Empfänger:innen in Betracht nimmt bzw. erfüllt (bspw. durch Service Customization, etwa durch Personalisierung von angebotenen Produkten oder individuelle Zusammenstellung von Serviceleistungen).

Human gestaltete Arbeit der Beschäftigten muss keineswegs eine Befriedigung der Bedürfnisse von DL-Empfänger:innen bedingen, auch wenn angenommen werden kann, dass human gestaltete Interaktionsarbeit sowohl den Beschäftigten als auch den DL-Empfänger:innen dient (vgl. Rieder, 2018). Eine integrierte Arbeits- und DL-Gestaltung, die nützlich für die Beschäftigten, die DLEmpfänger:innen und das DL-Unternehmen ist, stellt ein Defizit mit dringendem Forschungsbedarf im Sinne "gut gestalteter Koproduktion" von DL-Geber:innen und DL-Empfänger:innen dar. Für eine nachhaltige Gestaltung von Interaktionsarbeit reicht ein Nebeneinander der wissenschaftlichen Perspektiven nicht aus, um der Komplexität des Interaktionsnetzes bei DL gerecht zu werden (vgl. Rieder, 2018).

Durch die Verknüpfung von Arbeits- und DL-wissenschaft im Projekt SO-SERVE soll Interaktionsarbeit gleichermaßen menschengerecht und effizient gestaltet werden. Die Perspektiven der DL-Geber:innen und DL-Empfänger:innen wer- 
den gleichermaßen einbezogen, genauso wie die der weiteren, an der DL beteiligten, Akteur:innen. Innerhalb des interdisziplinären Ansatzes sollen nicht nur die Besonderheiten der Interaktionsarbeit selbst, sondern auch die Gestaltungmöglichkeiten beider Disziplinen berücksichtigt werden. Maßnahmen für die DL-Gestaltung bei Interaktionsarbeit schließen neben arbeitswissenschaftlichen Maßnahmen auch technologische Entwicklungen ein, um die durch den technologischen Fortschritt freiwerdenden Potenziale in der Interaktion sinnvoll wiedereinzusetzen. Auf diese Weise möchte das Projekt SO-SERVE das Ziel "gute Arbeit bei guten Dienstleistungen" oder "gute Dienstleistung bei guter Arbeit" erreichen.

Ziel des vorliegenden Whitepapers ist es, die Synergiepotentiale von Arbeitswissenschaft und Service Engineering aufzuzeigen und einen aktuellen Überblick über Ansätze zur Analyse und Gestaltung von Interaktionsarbeit in personenbezogenen $D L$ zu geben. 
werden. Human gestaltete Interaktionsarbeit beinhaltet, dass sie menschengerecht und menschenwürdig ausgeführt werden kann und damit die physische und psychische Gesundheit weder kurz- noch langfristig beeinträchtigt, sondern bestenfalls fördert und stärkt.

Bei der arbeitswissenschaftlichen Analyse und Bewertung von Arbeit stehen objektivierbare Arbeitsanforderungen (Arbeitsbedingungen) im Vordergrund. Daher folgt auch die Reihenfolge von Gestaltungsmaßnahmen einer Maßnahmenhierarchie: Zunächst werden bedingungsbezogene/verhältnisbezogene Maßnahmen umgesetzt (z. B. technische, organisatorische Maßnahmen), die durch personenbezogene/verhaltensbezogene Maßnahmen (z. B. Qualifizierung, Unterweisung) ergänzt werden können (vgl. §4 ArbSchG und TOP-Prinzip).

Das Service Engineering ist eine Teildisziplin der DL-Forschung und befasst sich mit der Entwicklung und Optimierung von DL. Ein Ziel des Service Engineering ist die Optimierung von DL-Systemen, durch die Schaffung von effizienten Strukturen und (wiederholbaren) Abläufen (Modularisierung) (vgl. Meyer \& Zinke, 2018). Dafür bedient sie sich bei Ansätzen aus der (industriellen) Produktentwicklung und nimmt die DL als zu entwickelndes „Produkt" in den Fokus („Productization“ (vgl. Baines et al., 2007) /"Modularization“ (vgl. Gremyr et al., 2019)).

Das Service Engineering ist, wie auch die DL-Forschung generell, aus verschiedenen Disziplinen gewachsen und hat eine interdisziplinäre Forschungsperspektive, jedoch mit einem starken Einfluss aus der Ingenieurswissenschaft worauf auch der Namensteil „Engineering“ hindeutet. Als Forschungsdisziplin wurde sie hauptsächlich in Deutschland vorangetrieben und ist definiert als Konzept zur systematischen Entwicklung und Gestaltung von DL unter Zuhilfenahme geeigneter Vorgehensmodelle, Methoden und Werkzeuge (vgl. Bullinger et al., 2003). DL werden dabei typischerweise auf drei Ebenen betrachtet. Diese sind die Potenzial-, Prozess- und Ergebnisdimension (vgl. Fähnrich \& Opitz, 2006). Hinzu kommt die Dimension des Marktes als phasenübergreifende Gestaltungsdimension (vgl. Bullinger \& Schreiner, 2006). Die zur Erbringung der DL notwendigen Prozesse werden schematisch abgebildet, $d$. h. in Form von Teilprozessen der DL als Vorgänge modelliert (d. h. strukturiert, 
beschrieben und visuell dargestellt) und in zyklischen Verfahren optimiert. In der Entwicklung und Verbesserung der Dienstleitung spielt auf Ebene der Prozessdimension die Intensität der Integration der Kund:innenperspektive eine zentrale Rolle (vgl. Haab et al., 2019).

Zusammenfassend beschäftigen sich Arbeitswissenschaft und Service Engineering gleichermaßen mit der Gestaltung von DL - wenn auch mit einem unterschiedlichen Fokus auf das Arbeits- bzw. das DL-System. Das verbindende Element zwischen Arbeitswissenschaft und Service Engineering stellen die Prozesse dar, durch welche die Akteur:innen des DL-Systems mittels Interaktionsarbeit die DL erstellen. Die Synergiepotenziale des aus Service Engineering und Arbeitswissenschaft entstehenden Social Service Engineering sind in Tabelle 1 dargestellt. 


\begin{tabular}{|c|c|c|c|}
\hline & $\begin{array}{c}\text { Service } \\
\text { Engineering }\end{array}$ & $\begin{array}{c}\text { Social Service Engine- } \\
\text { ering (SSE) }\end{array}$ & $\begin{array}{c}\text { Arbeits- } \\
\text { wissenschaft }\end{array}$ \\
\hline Leitbild & $\begin{array}{l}\text { Gute Dienstleis- } \\
\text { tungen }\end{array}$ & $\begin{array}{c}\text { Gute Arbeit bei guten } \\
\text { Dienstleistungen }\end{array}$ & Gute Arbeit \\
\hline $\begin{array}{l}\text { Zentrale } \\
\text { Gegen- } \\
\text { stände }\end{array}$ & $\begin{array}{c}\text { Prozesse, Ressour- } \\
\text { cen, Leistungsele- } \\
\text { mente }\end{array}$ & $\begin{array}{l}\text { systematische Ko-Ge- } \\
\text { staltung von Syste- } \\
\text { men, Prozessen und } \\
\text { Arbeitsanforderungen }\end{array}$ & $\begin{array}{l}\text { Menschen und In- } \\
\text { teraktionen im Kon- } \\
\text { text der MTO-Triade } \\
\text { (Mensch-Technik- } \\
\text { Organisation) }\end{array}$ \\
\hline $\begin{array}{l}\text { Primäre } \\
\text { Gestal- } \\
\text { tungs- } \\
\text { ziele }\end{array}$ & $\begin{array}{l}\text { Hohe Qualität und } \\
\text { hohe Produktivität }\end{array}$ & $\begin{array}{l}\text { Gesunde Arbeit als } \\
\text { Bedingung für effek- } \\
\text { tive und effiziente } \\
\text { Dienstleistungen }\end{array}$ & $\begin{array}{l}\text { Arbeits- und Ge- } \\
\text { sundheitsschutz, } \\
\text { humane Arbeitsge- } \\
\text { staltung }\end{array}$ \\
\hline $\begin{array}{l}\text { Primäre } \\
\text { Ziel- } \\
\text { gruppe }\end{array}$ & $\begin{array}{c}\text { Anbieter, Kund:in- } \\
\text { nen, Markt }\end{array}$ & $\begin{array}{l}\text { Zusammenspiel von } \\
\text { Anbieter, Beschäftig- } \\
\text { ten und Nutzer:innen }\end{array}$ & $\begin{array}{l}\text { Beschäftigte mit ih- } \\
\text { ren Arbeitsanforde- } \\
\text { rungen }\end{array}$ \\
\hline $\begin{array}{l}\text { Innovati- } \\
\text { onsver- } \\
\text { ständnis }\end{array}$ & $\begin{array}{l}\text { Primat der Tech- } \\
\text { nik / technische In- } \\
\text { novationen }\end{array}$ & $\begin{array}{l}\text { Technische und sozi- } \\
\text { ale Innovationen für } \\
\text { den Menschen }\end{array}$ & $\begin{array}{c}\text { Primat des Men- } \\
\text { schen / soziale In- } \\
\text { novationen }\end{array}$ \\
\hline $\begin{array}{l}\text { Gestal- } \\
\text { tungs- } \\
\text { zeitpunkt }\end{array}$ & eher prospektiv & $\begin{array}{l}\text { idealerweise } \\
\text { prospektiv }\end{array}$ & $\begin{array}{c}\text { in der Praxis häufig } \\
\text { korrektiv }\end{array}$ \\
\hline $\begin{array}{l}\text { Gestal- } \\
\text { tungsan- } \\
\text { sätze }\end{array}$ & $\begin{array}{l}\text { Technik-, ingeni- } \\
\text { eurwissenschaft- } \\
\text { lich, informatik- } \\
\text { zentrierte Ansätze }\end{array}$ & $\begin{array}{l}\text { Interdisziplinärer } \\
\text { Ansatz }\end{array}$ & $\begin{array}{c}\text { psychologische, so- } \\
\text { zialwissenschaftlich- } \\
\text { pädagogisch } \\
\text { zentrierte Ansätze }\end{array}$ \\
\hline
\end{tabular}




\section{Interaktionsarbeit als Aspekt von Dienstleistungen}

Im Projekt SO-SERVE wird eine Definition von Interaktionsarbeit verwendet, die einerseits an bestehende Definitionen von DL anknüpft und andererseits die Spezifik von Interaktionsarbeit im Vergleich zum Oberbegriff der DL-Tätigkeiten verdeutlicht.

Interaktionsarbeit stellt eine besondere Arbeitsform dar, die in unterschiedlichem Ausmaß im Rahmen von DL erbracht wird. DL sind nach Meyer (1991, zit. nach Fähnrich \& Opitz, 2006, S. 94) „angebotene Leistungsfähigkeiten, die direkt an externen Faktoren (Menschen oder deren Objekte) mit dem Ziel erbracht werden, an ihnen gewollte Wirkungen (Veränderung oder Erhaltung bestehender Zustände) zu erreichen."

DL beinhalten neben interaktiven Tätigkeitsbestandteilen an und mit Menschen (Interaktionsarbeit) immer auch monologische Teiltätigkeiten ohne erforderlichen Kontakt zu anderen Menschen (z. B. Arbeitsplanung, Berichterstattung, Dokumentation) (vgl. Hacker, 2009). Analog zu Hackers (2009) Unterscheidung von interaktiven und monologischen Tätigkeitsbestandteilen unterteilt Leimeister (2020) die DL-Erstellung in die Vorkombination und die Endkombination. Erst in der Endkombination, wenn der Service an oder mit der DL-nehmenden Person ausgeführt wird, findet Interaktionsarbeit statt.

Das Mischungsverhältnis aus interaktiv-dialogischen und monologischen Teiltätigkeiten ist für die Beschäftigten anforderungsbestimmend. Der wachsende Anteil monologischer Teiltätigkeiten widerspricht dabei häufig der Berufsphilosophie der Beschäftigten, die diese Arbeit vordergründig gewählt haben, „um mit Menschen arbeiten zu können“. Darüber hinaus gibt es Hinweise, dass die monologischen Teiltätigkeiten zum Teil als weniger anregend und beanspruchender erlebt werden als die interaktive Arbeit an DL-Empfänger:innen (vgl. Hacker, 2009).

In der ganzheitlichen Betrachtung von Arbeitssystemen und DL-Systemen hat sich zudem ein systemisches Verständnis durchgesetzt. Nach Böttcher (2009, S. 18) ist ein DL-System definiert als „[...] ein Konglomerat unterschiedlicher Akteure und Ressourcen [...], welche aufgrund ihrer Interaktion eine Zustandsän- 
derung zur Folge haben. [...] Diese Zustandsveränderung definiert die Funktionalität (respektive Dienstleistung) und beinhaltet einen Mehrwert für den Nachfrager und Initiator der Dienstleistungserbringung."

Da Interaktionsarbeit ein Teil von DL ist, gelten für sie die grundlegenden Merkmale von DL (in Anlehnung an DIN-Fachbericht 75: 1998: Service EngineeringEntwicklungsbegleitende Normung (EBN) für Dienstleistungen):

- Immaterialität: Im Ergebnis der Interaktionsarbeit gibt es oftmals kein "greifbares", physisches Produkt. Dies hat nicht nur Auswirkungen auf die Messbarkeit der DL-Ergebnisse von Interaktionsarbeit, sondern auch auf Aspekte wie Anerkennung und Wertschätzung dieser Erwerbsarbeitsform.

- Zeitpunkt der Herstellung: Die Arbeitsergebnisse von Interaktionsarbeit werden in dem Augenblick "konsumiert", in dem sie "hergestellt" werden (uno-actu-Prinzip). Eine DL-Erstellung „auf Lager" ist nicht möglich, was Auswirkungen auf die Personal- und Kapazitätenplanung haben kann. Darüber hinaus erfordert die Interaktion die Integration der DL-Empfänger:innen und ggf. DL-Nehmer:innen.

- Kund:innenkontakt/Integration eines externen Faktors: Bei DL sind die DLEmpfänger:innen oft stark in den Leistungserstellungsprozess involviert bzw. sie sind Teil dieses Prozesses und wirken an der Leistung mit. Bei Interaktionsarbeit ist die Kooperation der DL-Empfänger:innen sogar unerlässlich. Es ist Teil des Arbeitsauftrages der Beschäftigten, die erforderliche Mitarbeit des „Arbeitsgegenstandes“ selbst zu erzeugen (vgl. Rieder \& Voß, 2009).

- Prozessorientierung: Der Leistungserstellungsprozess stellt häufig die eigentliche DL dar, DL-Empfänger:innen bzw. DL-Nehmer:innen kaufen daher gewissermaßen einen Vorgang bzw. einen Prozess. Insbesondere bei der Arbeit an und mit Menschen ist dieser Prozess mit Unbestimmtheiten und Unwägbarkeiten verbunden, weil er durch die DL-Empfänger:innen bzw. DL-Nehmer:innen mitgestaltet wird. Daraus ergeben sich 
Grenzen für die Planbarkeit des Prozesses, sowohl aus Sicht der DL-Geber:innen, als auch aus Sicht des Service Engineering im Sinne der Standardisierbarkeit.

Durch die notwendige Kollaboration mit den DL-Empfänger:innen sind DL grundsätzlich von Interaktion geprägt (vgl. Baumgärtner \& Bienzeisler, 2006). Interaktion wird in diesem Zusammenhang als jeglicher (unmittelbarer und mittelbarer) Kontakt zwischen DL-Geber:innen und DL-Empfänger:innen beschrieben. Der Ausdruck „personennahe DL“ bezeichnet diejenigen $\mathrm{DL}$, die besonders stark von Interaktion geprägt sind - wie bspw. Pflegetätigkeiten, kosmetische DL oder Bildung. Die DL-Gestaltung im Service Engineering konzentriert sich jedoch dabei mehr auf die zu entwickelnden und optimierbaren Prozesse, als auf schwer fassbare zwischenmenschliche Faktoren wie Beziehungsqualität, Emotionen und Hierarchien (ebd.). Arbeiten, die Interaktionsarbeit aus Service-Engineering-Perspektive thematisieren, bedienen sich den Definitionen, die in Soziologie oder Arbeitswissenschaft entwickelt wurden, wie z. B. Haab et al. (2019) und Leimeister (2020). Eine eigene Definition von Interaktionsarbeit existiert im Service Engineering bislang nicht.

Hacker (2009) nutzt für seine Ausführungen zur Interaktionsarbeit die Bezeichnung "dialogisch-interaktive Erwerbsarbeit". Dialogisch betont die wichtige Rolle der Sprache als Instrument von DL-Geber:innen, um die Interaktion mit DL-Empfänger:innen zu steuern. Der sprachliche Informationsaustausch stellt einen leistungsbestimmenden Bestandteil der Arbeit an und mit Menschen dar. Der Begriff interaktiv „ergänzt die möglichen nichtsprachlichen Anteile und das wechselseitige Aufeinanderwirken von Individuen zur Verhaltensabstimmung" (ebd., S. 17).

Interaktionsarbeit ist ein Tätigkeitsbestandteil nahezu jeder DL, die eine Interaktion zwischen DL-Geber:innen und DL-Empfänger:innen beinhaltet. Es ist jedoch zu unterscheiden "zwischen der Interaktionsarbeit als Kern der Tätigkeit, so wie dies überwiegend bei personenbezogenen Dienstleistungen der Fall ist, und der Interaktionsarbeit als unvermeidbarem und notwendigem Bestandteil gegenstands- und sachbezogener Dienstleistungen“ (Böhle \& Weihrich, 2020, S. 14). Personenbezogene DL werden beispielsweise in der Gesundheitsversor- 
gung und im Bildungswesen angeboten. Zu den sachbezogenen DL zählen unter anderem Reparatur- und Transportleistungen. Insbesondere das Beispiel produktbegleitender DL (z. B. die produktbezogene Beratung, Customization, Wartung) macht deutlich, dass zunehmend auch die Interaktion bei sachbezogenen DL leistungsbestimmend ist.

Interaktionsarbeit ist mit Besonderheiten im Arbeitshandeln der DL-Geber:innen verbunden. Hacker (2009) weist darauf hin, dass der "Arbeitsgegenstand" bei dialogisch-interaktiver Arbeit ein Individuum bzw. Subjekt (DL-Empfänger:in) ist, welches eigene Ziele und Wünsche hat und nicht "bearbeitet" werden kann wie ein Objekt. Je nach Art der DL (z. B. Haarschnitt, Diätassistenz) ist ein unterschiedliches Maß an Koproduktion erforderlich, d. h. die Mitwirkung der DL-Empfänger:innen an der DL-Erstellung. Aufgabe von DL-Geber:innen ist es, die Ziele und Wünsche der DL-Empfänger:innen zu berücksichtigen und die Koproduktion zu gestalten. Bedingt durch die Subjekt- und Koproduzent:innenrolle der DL-Empfänger:innen ist das Handeln von DL-Geber:innen nur begrenzt planbar. Eine weitere Arbeitsanforderung der DL-Geber:innen ist der Umgang mit den Emotionen der DL-Empfänger:innen (z. B. Motivieren von Schüler:innen, Beruhigen von Patient:innen). Darüber hinaus ist es häufig erforderlich, dass DL-Geber:innen ihre eigenen Gefühle unterdrücken müssen (z. B. Ärger, Ekel), aber geforderte, regelgerechte Gefühle wie Freude zeigen müssen (ebd.).

Diese Besonderheiten von Interaktionsarbeit greifen Böhle, Stöger und Weihrich (2015) in ihrem integrativen „Konzept der Interaktionsarbeit" auf. Das Konzept besteht aus vier Komponenten: 1. Kooperationsarbeit (Herstellung einer Kooperationsbeziehung zwischen DL-Geber:innen und DL-Empfänger:innen), 2. Emotionsarbeit (Umgang mit den eigenen Emotionen aus Sicht der DL-Geber:innen ), 3. Gefühlsarbeit (Umgang mit bzw. Beeinflussung der Gefühle der DL-Empfänger:innen) und 4. Subjektivierendes Arbeitshandeln (Umgang mit Unwägbarkeiten und Grenzen der Planung).

Eine weitere Besonderheit von Interaktionsarbeit besteht darin, dass der Nutzen von Interaktionsarbeit über den reinen "Geldwert" hinausgeht. Neben der Interaktion selbst, die als eigenständiger Wert betrachtet werden kann, sowie dem funktionalen Nutzen für DL-Empfänger:innen (Value in Use), spielen bei 
der Betrachtung von Interaktionsarbeit sowohl der emotionale als auch der soziale Wert eine Rolle - dies gilt für DL-Geber:innen ebenso wie für DL-Empfänger:innen. Als relevante nicht-monetäre Werte sind bspw. die Gesundheit und Weiterentwicklung der Beschäftigten, eine gute Beziehungsqualität. Reputation/Alleinstellungsmerkmal für das Unternehmen, Zufriedenheit, Inspiration sowie positive ökologische Auswirkungen zu nennen. Ein „erweiterter Qualitätsrahmen“ für Interaktionsarbeit sollte nicht nur Ziel-Standards (z. B. Erfüllung monetärer Leistungsindikatoren, Erfüllung der Erwartungen von DL-Empfänger:innen), sondern auch Handlungsstandards (z. B. Schaffen von Handlungsspielräumen, Gewährleistung der Weiterentwicklung der Beschäftigten) beinhalten.

Eine Forschungslücke besteht derzeit in der Gestaltung von Interaktionsarbeit, welche nicht nur die oben beschriebenen Besonderheiten von Interaktionsarbeit berücksichtigt, sondern gleichzeitig auch Effizienz- und Qualitätskriterien aus Sicht der DL-Empfänger:innen und DL-Nehmer:innen einbezieht. Derzeit berücksichtigt nur die Arbeitswissenschaft die besonderen Arbeitsanforderungen der Interaktionsarbeit für die Beschäftigten. Die Interaktion bei der DL-Erstellung wird im Service Engineering bisher nur unzureichend betrachtet (vgl. Haab et al., 2019). Die schematische und formalisierende Herangehensweise des Service Engineering erschwert die Abbildung von Aspekten, die bei der Interaktionsarbeit relevant sind, wie bspw. Emotionen, Hierarchien und Beziehungen. Aufgrund der eher technischen Herangehensweise sind auch Methoden zur Abbildung und Gestaltung von Interaktionsarbeit im Service Engineering kaum vorhanden (vgl. ebd.). Zudem sind diese Aspekte der Interaktionsarbeit größtenteils situationsbedingt und personenbezogen, was dem SE-eigenen Standardisierungsstreben teilweise entgegenläuft.

Die Verknüpfung von Arbeitswissenschaft und Service Engineering kann nicht nur die Reichweite und Akzeptanz von menschzentrierten Gestaltungsmaßnahmen in Unternehmen erhöhen, sondern auch zu einer ganzheitlichen Beantwortung wirtschaftlicher Fragestellungen aus Unternehmen beitragen. 


\section{Besonderheiten der Dienstleistungen Pflege und Kinderbetreuung}

Die im Projekt SO-SERVE ausgewählten DL-Bereiche der Pflege und Kinderbetreuung unterscheiden sich von anderen Formen personenbezogener DL in verschiedenen Punkten:

- Dauer und Intensität der DL-Beziehung

Die DL der ambulanten Pflege und der Kinderbetreuung sind geprägt von einer länger andauernden und intensiven Interaktionsbeziehung. Die Interaktionsarbeit findet dabei nicht im Rahmen von ein- oder mehrmaligen, jeweils in sich abgeschlossenen DL-Prozessen statt, sondern geschieht fortlaufend über einen längeren Zeitraum.

- DL-Nehmer:in und DL-Empfänger:in müssen nicht dieselbe Person sein

In der Pflege und in der Kinderbetreuung besteht eine Besonderheit darin, dass DL-Empfänger:in und DL-Nehmer:in oftmals nicht zusammenfallen. So sind es bspw. die Eltern, die als DL-Nehmer:innen die Betreuungsleistung in Anspruch nehmen und dafür bezahlen, aber die eigentlichen DL-Empfänger:innen sind die Kinder. Aus dieser besonderen Konstellation können Konflikte entstehen, wenn Bedürfnisse oder Wünsche von DL-Nehmer:innen und DL-Empfänger:innen divergieren, z. B. die Bedürfnisse der betreuten Kinder und Wünsche von Erziehungsberechtigten oder die Interessen der Pflegebedürftigen und Forderungen von Angehörigen. Hier stellt sich die heikle Frage, wem die DL-Geber:innen „dienen“ und wie die Anforderungen von DL-Geber:innen, DL-Nehmer:innen und DL-Empfänger:innen zusammengebracht werden können.

- Größe des Interaktionsnetzwerkes

In der Pflege und der Kinderbetreuung geht die Interaktionsarbeit weit über die Interaktion zwischen DL-Geber:innen und DL-Empfänger:innen hinaus. 


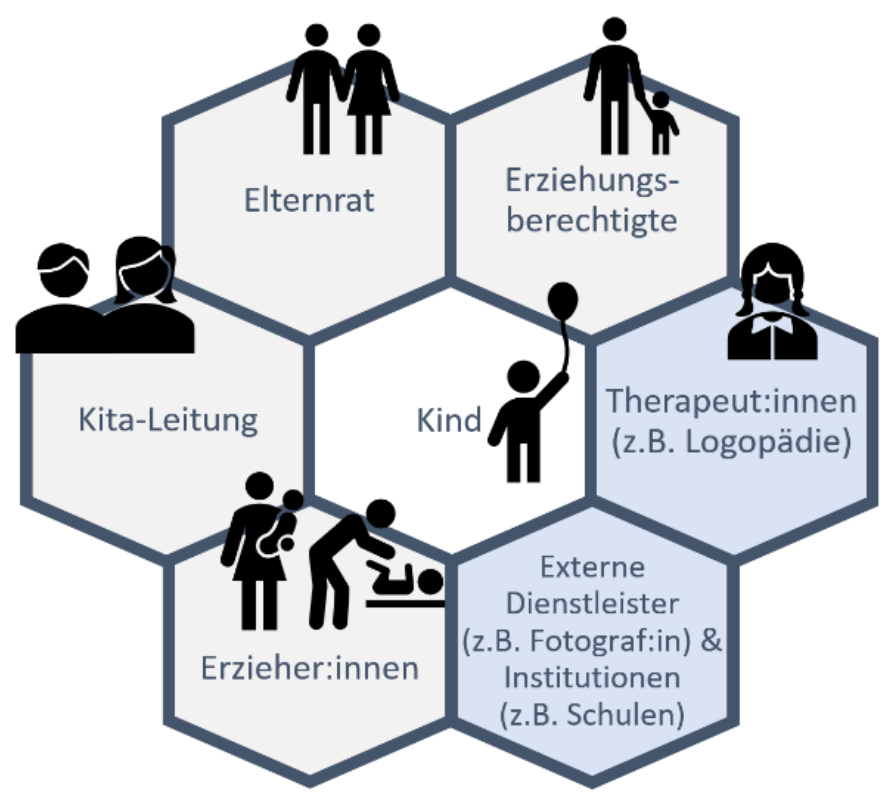

Abbildung 3: Stakeholder Map Interaktionsarbeit in der Kita

Im Falle der Kinderbetreuung umfasst das Interaktionsnetz, wie in Abbildung 3 dargestellt, eine Vielzahl von Personen, die im Sinne einer Bildungs- und Erziehungspartnerschaft miteinander interagieren. Neben dem betreuten Kind sind das dessen Erziehungsberechtigte und weitere Personen aus dem persönlichen Umfeld des Kindes, ein Erzieher:innen-Team, die Kita-Leitung (grau dargestellt) sowie weitere seitens der Kita involvierte Personen und Institutionen (blau dargestellt). Ähnlich verhält es sich bei der DL der ambulanten Pflege. Auch hier stehen Patient:innen und Pflegekraft bzw. Pflegeteam im Zentrum der DL-Tätigkeit. Beteiligt an der Interaktionsarbeit sind aber ebenfalls Angehörige, die mit dem Pflegedienst in Verbindung stehen und deren Handeln (Pflege) sich auf die DL auswirkt, sowie Externe (z. B. Ärzt:innen). Das Interaktionsnetzwerk existiert innerhalb eines sogenannten STEEP-Kontexts (STEEP - social, technological, economic, environmental, political) (vgl. Freund \& Spohrer, 2013). Der Erfolg und die Qualität der Interaktionsarbeit ist auch vom Kontext, in dem das Netzwerk agiert, abhängig.

- Besonderheiten der Finanzierung / Abhängigkeit von externen Regelungen

Aus dem Sozialstaatsprinzip und der daraus abgeleiteten Fürsorgepflicht des Staates ergibt sich, dass die DL in der Pflege und der Kinderbetreuung solidargemeinschaftlich und damit öffentlich (anteils-)finanziert werden. Die daraus resultierende Leistungs- und Kostenträgerstruktur schafft Rahmenbedingungen, die sowohl in der Pflege als auch in der Kinderbetreuung zu einem Dilemma aus Ökonomisierungsdruck, individuellen (dienstleisterseitigen) und auch gesellschaftlichen Ansprüchen führen (vgl. Hielscher et al., 2013). Die Kostenträgerstruktur führt dazu, dass Dienstleister nicht frei 
in der Preisgestaltung ihrer DL sind, was den finanziellen und personellen Spielraum für Innovationen einschränken kann.

Im Pflegebereich besteht neben Personalknappheit ein hoher Kostendruck. Es können gegenüber dem Kostenträger nur rein pflegerische Tätigkeiten abgerechnet werden, die engen Zeitvorgaben unterliegen und dokumentiert werden müssen. Zudem ist jede Änderung der geplanten Pflegeleistungen zusätzlich rechtfertigungs- und dokumentationspflichtig. Oft bleibt dadurch zu wenig Zeit für menschlichen Beistand, respektvolle Beratung oder eine angemessene Aktivierung der Patient:innen (Hacker, 2009; Hielscher et al., 2013). Ähnliches gilt für die Kinderbetreuung, in der sich der Charakter der Interaktion durch zeitgleich oder zusätzlich durchzuführende Dokumentations- und Beobachtungsaufgaben verändert (Hielscher et al., 2013).

Das Gelingen der Interaktionsarbeit ist darüber hinaus auf zahlreiche Begleitprozesse im Rahmen der Eltern-/ bzw. Angehörigenarbeit und Teamarbeit angewiesen. Dabei handelt es sich in erster Linie um Aushandlungs-, Abstimmungs-, Kommunikations- und Koordinationsprozesse im Interaktionsnetzwerk. Obgleich dies elementare Bestandteile der DL-Tätigkeit sind und Einfluss auf die DL-Qualität haben, sind sie kaum greifbar und damit letztlich auch schwer ökonomisierbar. 


\section{Technik und Digitalisierung bei der Gestaltung von Dienstleistungen}

Besonders weil das Digitalisierungsgeschehen im DL-Bereich weiterhin an Fahrt gewinnt (vgl. Thorein \& Riesenberg-Mordeja, 2018), ergibt sich die Chance, gestalterisch die digitale Transformation in der Interaktionsarbeit zu begleiten und prospektiv zu lenken. Angesichts rasanter technischer Innovationen und sich dadurch stetig ändernden Anforderungen ist es hierbei notwendig, Risiken und Befürchtungen aber auch Potenziale und Chancen gleichermaßen in den Blick zu nehmen.

Aus arbeitswissenschaftlicher Sicht ist die Digitalisierung eine mögliche Gestaltungsmaßnahme, aber kein unerlässliches Gestaltungsziel. Die DL-Wissenschaft dagegen nutzt die Technologisierung und Digitalisierung als „Mittel der Wahl", um Prozesse neu zu gestalten und neue Potentiale zu erschließen. Mit Hilfe der technischen und digitalen Möglichkeiten können Beschäftigte bei ihrer interaktiven Arbeit psychisch und physisch entlastet und die DL-Qualität sowohl für die DL-Empfänger:innen (z. B. Patient:innen, Kinder) als auch für die DL-Nehmer:innen (z. B. Angehörige und Erziehungsberechtigte) erhöht werden (vgl. Hülsken-Giesler, 2015; Hülsken-Giesler \& Daxberger, 2018). Im besten Fall werden Fehlbeanspruchungen und deren Folgen durch die technischen Möglichkeiten verringert. Die Technik kann eine gute Interaktionsbeziehung zwischen den Beschäftigten und DL-Empfänger:innen unterstützen, indem sie Zeit für den Kern der Interaktionsarbeit schafft.

Durch die digitalen und technischen Innovationen entstehen bereits heute komplett neue DL, z. B. im Bereich des stationären und Online-Handels, und innovative Möglichkeiten für die DL-Entwicklung. Für die DL-Gestaltung bedeutet die Digitalisierung auch, dass existierende Prozesse und klassische Geschäftsmodelle hinterfragt und ggf. angepasst werden müssen. Ein Beispiel für sich wandelnde Teilprozesse von DL sind die Beratungen in Reisebüros oder im Einzelhandel, welche zunehmend von digitalen Formaten wie crowd-basierten Produktbewertungen verdrängt oder von Chat-Bots übernommen werden.

Die dem Service Engineering zugrundeliegende Idee, DL wie „Produkte“ zu gestalten, muss sich im Zuge der Digitalisierung neuen Anforderungen stellen. Modelle aus der Industrie entsprechen nicht länger den dynamischen und 
schnell wachsenden Anforderungen agiler DL-Entwicklung, welche sich durch individuelle Kund:innenorientierung und Flexibilität auszeichnet. Im Hinblick auf personenbezogene DL ist in diesem Zusammenhang der junge Ansatz des Service Engineering Plus zu nennen. Service Engineering Plus kombiniert Methoden des Service Engineering mit partizipativen Ansätzen, um technikgetriebene Innovation personenbezogener DL kundschaftsgerecht zu realisieren. Konkret bedeutet das, dass DL-Nehmer:innen bereits frühzeitig in den Innovationsprozess einbezogen werden und innovative Ansätze zur Neugestaltung der DL in Form von Prototypen evaluieren (vgl. Menschner et al., 2014).

Zu den Risiken der Digitalisierung und des Technikeinsatzes zählen Phänomene wie Verdichtung der Arbeit, Entgrenzung von Arbeit und Freizeit sowie Spezialisierungs- und De-Qualifizierungsprozesse (vgl. Hacker, 2018b; Rieder, 2018; Shire \& Leimeister, 2012). Technische Innovationen können auch das Ausmaß an persönlicher Zuwendung verringern, was sowohl durch die DL-Geber:innen als auch die DL-Empfänger:innen als unbefriedigend empfunden werden kann. Die DL-Geber:innen erleben möglicherweise Diskrepanzen zu ihrem Berufsrollenbild („Ich möchte eigentlich mit Menschen arbeiten“) und die DL-Empfänger:innen entwickeln im ungünstigsten Fall das Gefühl, „wie ein Gegenstand behandelt zu werden" (Entmenschlichung bzw. Desubjektivierung). Weitere Risiken aus der Perspektive der DL-Geber:innen in bestehen im Missbrauch der Technik als Kontrollinstrument, in der Enteignung von Wissen sowie im Verlust von Autonomie (vgl. Bleses et al., 2018; Widuckel, 2015).

Bei allen Digitalisierungsbestrebungen muss auch in der zukünftigen DL-Entwicklung grundsätzlich berücksichtigt werden, dass Interaktionsarbeit dem urmenschlichen Bedürfnis von DL-Empfänger:innen und DL-Geber:innen nach sozialer Interaktion, Integration und Beziehung entsprechen muss, und nicht durch Entmenschlichung der Interaktion zur Selbst-„,bedienung“ bzw. zur Mensch-Automaten-,Interaktion“ degradiert werden sollte (vgl. Bleses et al., 2018; Hacker, 2009). Technik soll als Unterstützung zeitliche Freiräume schaffen, durch die Beschäftigte in die Lage versetzt werden, menschenorientiert zu arbeiten und so den Ansprüchen der DL-Empfänger:innen und DL-Nehmer:innen sowie ihrem eigenen professionellen Selbstanspruch gerecht zu werden. Technische Lösungen dürfen demnach nicht ausschließlich auf Effizienzsteige- 
rung und Digitalisierung um ihrer selbst willen ausgerichtet sein, sondern müssen im Anwendungsfeld der Interaktionsarbeit stets mit höchster Sorgfalt und unter Berücksichtigung der individuellen Anforderungen von DL-Geber:innen, DL-Empfänger:innen und DL-Nehmer:innen eingesetzt werden. Somit sollte vor allem das Zusammenspiel gestaltet werden, das sich aus den technisierten und menschlichen Tätigkeiten ergibt. 


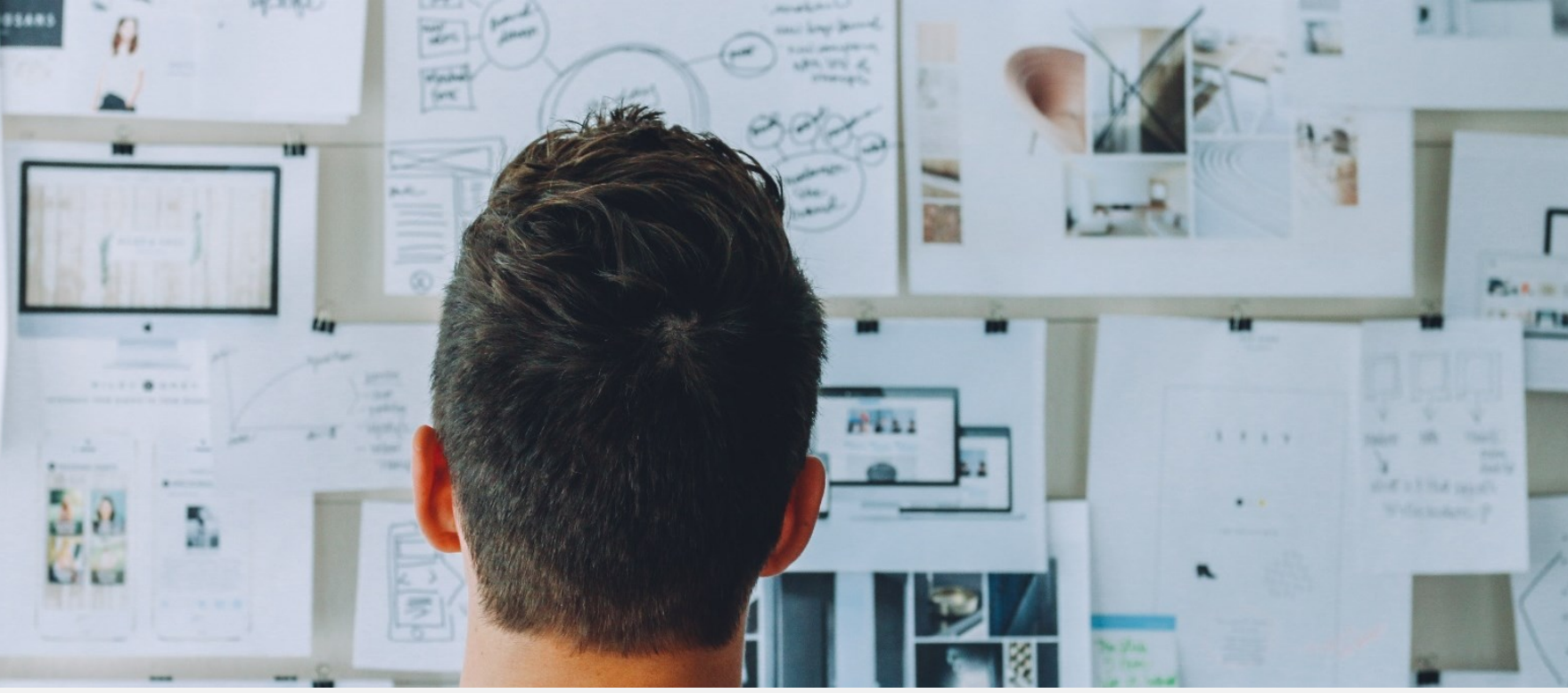

\section{Förderlandschaft Dienstleistungsgestaltung}

Co-Autor:innen des Kapitels: Bartetzko, Marit (ATB Arbeit, Technik und Bildung gGmbH); Freitag, Mike (Fraunhofer IAO); Peter, Christoph (Universität Kassel, ITEG); Pfahl, Sebastian (METOP GmbH); Robra-Bissantz, Susanne (TU Braunschweig); Sanders, Tobias (ATB Arbeit, Technik und Bildung gGmbH)

Mit zunehmender Bedeutung der DL-Branche beschäftigen sich auch immer mehr Forschungsprojekte mit der Gestaltung von DL im Allgemeinen und der Interaktionsarbeit im Speziellen. Um einen groben Überblick über die in der Förderlandschaft anzutreffenden Projekte zu geben, wurde in Abbildung 4 zunächst eine Übersicht der inhaltlichen Schwerpunkte einer Auswahl der in der Projektdatenbank zum Programm „Zukunft der Arbeit” (Stand 10.06.2021) zugänglichen Förderprojekte erstellt. Die Auswahl enthält alle Projekte der Bekanntmachungen "Arbeit an und mit Menschen", "Arbeit in der digitalisierten Welt", "Gesund ein Leben lang" sowie „Präventive Maßnahmen für die sichere und gesunde Arbeit von Morgen" und wurde um weitere Projekte ergänzt, die sich mit personenbezogener DL-Arbeit befassen.

Alle Projekte beziehen sich auf digitale Transformationsprozesse und die Gestaltung zukunftsgewandter Arbeitswelten. Dabei unterscheiden sich die Projekte in ihrem Fokus auf soziale versus technische Innovation sowie auf sachbezogene Arbeit vs. personenbezogene Arbeit:

- I. Quadrant: Projekte über soziale Innovationen bei sachbezogener Arbeit

(z. B. lernförderliche Arbeitsgestaltung in der Fertigung) 
- II. Quadrant: Projekte über soziale Innovationen im Kontext personenbezogener Arbeit (z. B. Trainingskonzepte zur Selbstentlastung für Pflegekräfte)

- III. Quadrant: Projekte über technikbezogene Innovationen für sachbezogene Arbeit (z. B. Einsatz digitaler Assistenzsysteme in der Produktion)

- IV. Quadrant: Projekte über technische Innovationen für personenbezogene Arbeit (z. B. Entwicklung eines datenbasierten Versorgungsalgorithmus für die Versorgung von Schlaganfallpatient:innen)

Ergänzt wurde die Übersicht um Projekte mit Bezug zur Arbeitsgestaltung in der Interaktionsarbeit aus weiteren Förderprogrammen des Bundes, z. B. „Innovation und Dienstleistung", „Forschung für Dienstleistung" sowie "Technologie und Dienstleistungen im demografischen Wandel“.

Die Darstellung macht deutlich, dass der Anteil derjenigen gelisteten Förderprojekte, die den Forschungsfokus auf die soziale Innovation, also die Gestaltung von Arbeitsprozessen und Rahmenbedingungen mittels methodisch konzeptioneller Ansätze legt (Quadrant I \& II), gegenüber Projekten mit Fokus auf technischen Innovationen überwiegt. Durch den Forschungsschwerpunkt „Arbeiten an und mit Menschen" sind zahlreiche Projekte vertreten, die ihren Schwerpunkt auf soziale und technische Innovation im Bereich der personenbezogenen Arbeit legen (Quadrant II und IV). Auch Projekte, die soziale oder technische Innovation branchenübergreifend oder branchenunabhängig betrachten (vertikale Achse), sind häufig Gegenstand der Förderung. 


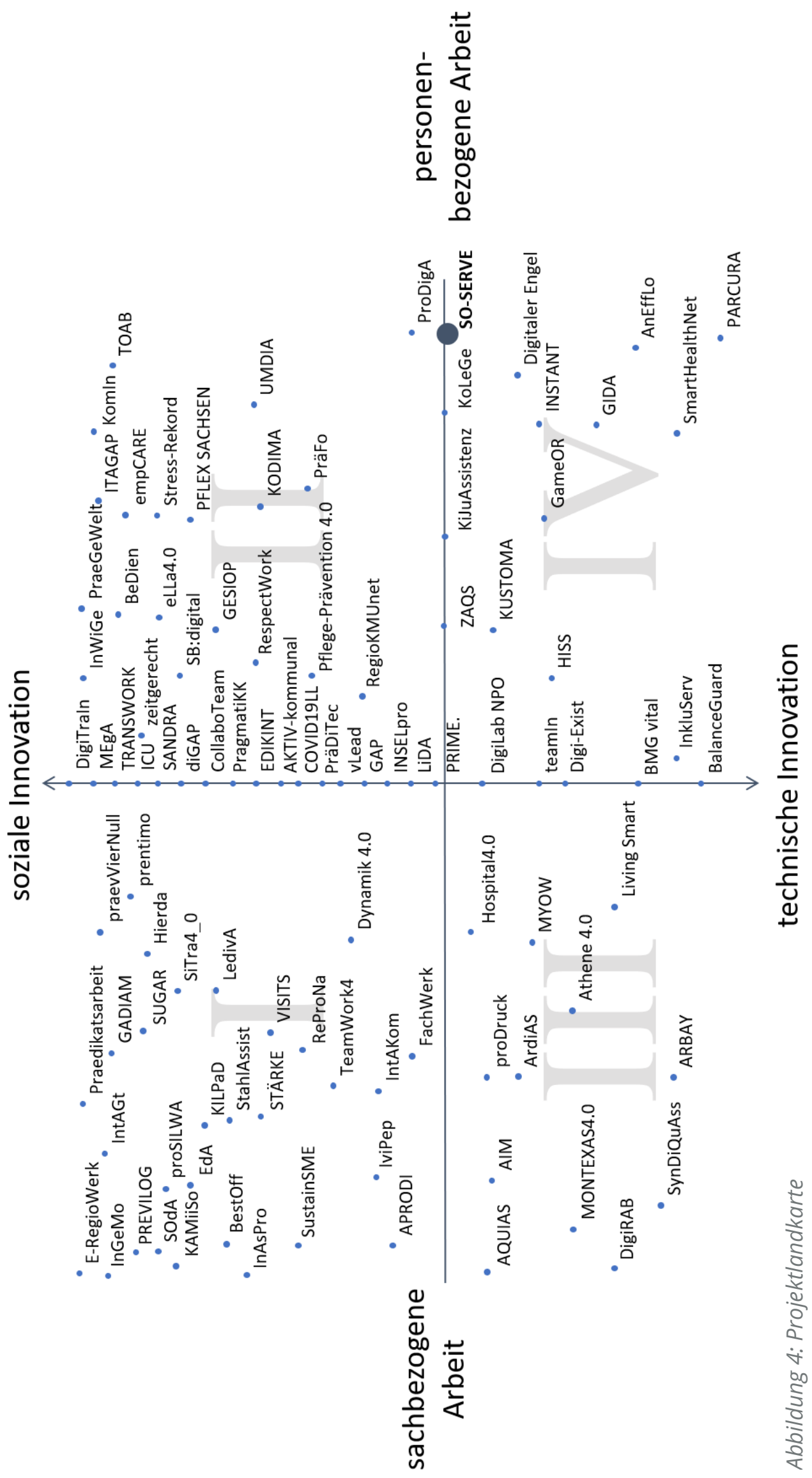


Die Gestaltung und methodische Unterstützung von Interaktionsarbeit werden von einer Reihe verschiedener Projekte adressiert. Einen Schwerpunkt bildet hier die Pflegebranche. So wird etwa im Projekt Digitaler Engel ein tragbares, digitales Assistenzsystem für Pflegekräfte entwickelt, um Interaktionsarbeit zwischen Pflegenden und Patient:innen mittels Bereitstellung von Informationen und Handlungsempfehlungen zu erleichtern. Um die Entwicklung digitaler Assistenzsystemen für Beschäftigte geht es auch in Projekten wie GIDA (Digitalisierungsstrategien für die Soziale Arbeit), KoLeGe (Kommunikations-, Informations- und Lernsoftware für die mobile Pflege), BalanceGuard (digitales Stressmonitoring) und HISS (Bot-Technologie zur Entlastung von Support-Dienstleister:innen).

Neben innovativen technischen Lösungen für bestehende Probleme im Rahmen der Interaktionsarbeit stehen auch methodische Ansätze der Arbeitsgestaltung im Fokus der Forschungsprojekte. Dabei werden etwa Auswirkungen von Unterbrechungen in digital gerahmter Interaktionsarbeit untersucht (UMDIA), Ansätze zur Gamification der Schichtdienstplanung in der stationären Altenpflege entwickelt (GamOR), Potentiale digitaler Unterstützung in partizipativen pädagogischen Arbeitsprozessen betrachtet (KiJuAssistenz) oder Lern- und Experimentierräume zur Verbesserung von Arbeitsprozessen in der ambulanten Pflege bereitgestellt (PFLEX SACHSEN). 


\section{Methoden zur Gestaltung von Interaktionsarbeit}

Co-Autor:innen des Kapitels: Freitag, Mike (Fraunhofer IAO); Kieffer-Radwan, Sonja (Fachhochschule des Mittelstands); Pfahl, Sebastian (METOP GmbH); Robra-Bissantz, Susanne (TU Braunschweig); Sanders, Tobias (ATB Arbeit, Technik und Bildung gGmbH)

Die interdisziplinäre Zusammenarbeit im Projekt SO-SERVE kombiniert die vorrangig arbeitsbedingungsbezogene, beschäftigtenorientierte Sicht der Arbeitswissenschaft mit der primär effizienz- und kundschaftsorientierten Sicht des Service Engineering. Beide Disziplinen bringen entsprechende Methoden zur Analyse und Gestaltung von Interaktionsarbeit in die Zusammenarbeit ein. Der Gestaltungsprozess muss, sowohl aus Sicht der Arbeitswissenschaft als auch aus Sicht des Service Engineering, als kontinuierlicher Begleitprozess verstanden werden (vgl. Bullinger \& Schreiner, 2006), der über den gesamten DL-Zyklus hinweg zur Anpassung an die Bedürfnisse der DL-Nehmer:innen und DLGeber:innen und damit zur kontinuierlichen Verbesserung der DL für alle Beteiligten beiträgt.

Bewährte Methoden der Arbeitswissenschaft haben sich, wie oben beschrieben, aus der Forschung in produzierenden Unternehmen entwickelt. Das Hauptaugenmerk der Methoden liegt auf der systematischen Analyse und Bewertung von Arbeitsprozessen hinsichtlich ihrer Güte (Bewertungskriterium: beeinträchtigungsfreie, lern- und persönlichkeitsförderliche Tätigkeiten, vgl. Hacker \& Richter, 1980) sowie auf der Gestaltung der Arbeitsbedingungen für DL-Geber:innen und deren Evaluation.

Die im Service Engineering eingesetzten Methoden dienen der Beschreibung und Gestaltung von DL auf Prozess-, Potenzial-, Ergebnis- und Marktebene. 
Zentral sind dabei Effizienz, Wiederholbarkeit und Qualität von DL (vgl. Meyer \& Zinke, 2018). Im Service Engineering liegt ein klarer Fokus auf dem Einsatz von Technologien als Innovationstreiber für DL. Der Mensch wird dabei vorrangig in seiner Rolle als Interaktionspartner:in betrachtet, wobei Interaktion hier primär die Mensch-Maschine-Interaktion meint, oder als Kund:in, dessen/deren Interesse am Produkt es zu wecken bzw. erhalten gilt.

Die Übertragung der Methoden auf die Arbeit an und mit Menschen ist mit vielen Herausforderungen verbunden. Ziel der interdisziplinären Zusammenarbeit ist es, für die Gestaltung von Interaktionsaktionsarbeit geeignete Methoden auszuwählen, zu beschreiben, (weiter) zu entwickeln und in einem Methodenbaukasten für Praktiker:innen bereitzustellen. 


\section{Expert:innenworkshop „Systematisch Human - Die Zukunft der Gestaltung von Interaktionsarbeit“}

Um die Methodenvielfalt mit breiter Expertise abzubilden und die Methodenauswahl für das Social Service Engineering zu unterstützen, veranstaltete das Projekt SO-SERVE am 19. März 2021 in Zusammenarbeit mit dem Forschungsnetzwerk FOKUS:SE und dem Unternehmensnetzwerk LESSIE einen Expter:innenworkshop. Im Rahmen dieses unter dem Titel „Systematisch Human - Die Zukunft der Gestaltung von Interaktionsarbeit" virtuell abgehaltenen Workshops waren die Expert:innen aus Arbeitswissenschaft und Service Engineering gleichermaßen aufgerufen, die von Ihnen in der Analyse und Gestaltung von DL genutzten Methoden auf einem digitalen Whiteboard, wie in Abbildung 5.

zu sehen, zusammenzutragen und zu diskutieren. Die Teilnehmenden, die sich dem Bereich Arbeitswissenschaft zuordneten, sammelten ihre Methoden auf grünen Kärtchen, die Teilnehmenden aus dem Bereich Service Engineering auf blauen Kärtchen.

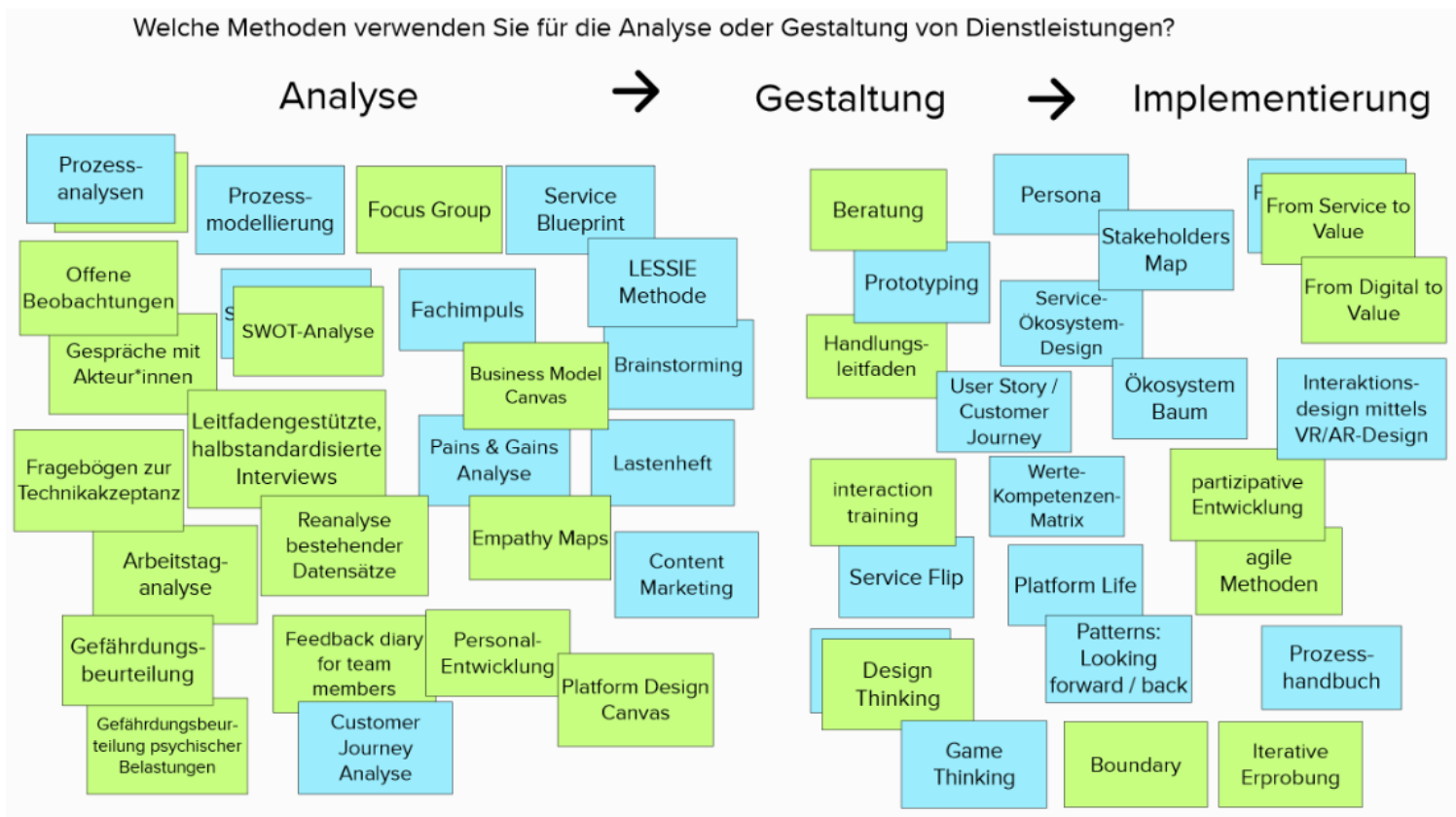

Abbildung 5: Brainstorming-Ergebnisse aus einer Session zu Methoden der Analyse und Gestaltung von $D L$ 
Im Laufe des Workshops wurden die den Sammlungsprozess strukturierenden Begriffe „Analyse“ und „Gestaltung" um den Begriff „Implementierung" erweitert. Diese Erweiterung wurde damit begründet, dass sich Service Engineering vornehmlich auf das Entwerfen und Testen von neuen technischen Lösungen bezieht und die Implementierung einen entsprechend großen Raum in der DLEntwicklung einnimmt. Neben der reinen technischen Realisierung digitaler Lösungen und dem praktischen Einsatz entwickelter Lösungen umfasst diese Phase auch die umfangreiche Überführung der Lösungen ins "Tagesgeschäft" von Unternehmen. Unter den Arbeitswissenschaftler:innen wurde der Begriff der Gestaltung dagegen weiter gefasst, sodass die Gestaltungsphase die Implementierung bereits inkludiert. Es wurde von Vertreter:innen beider Wissenschaftsdiziplinen darauf hingewiesen, dass in der Praxis besonders großer Unterstützungsbedarf in der Implementierungsphase (i. S. der Umsetzung von Gestaltungsmaßnahmen) besteht.

Die "klassischen“ Gestaltungsmethoden der Arbeitswissenschaft (z. B. Job Rotation, Pausengestaltung, Gestaltung der Arbeitsumgebung) waren auf dem Methoden-Board nicht vertreten, jedoch wurden von Arbeitswissenschaftler:innen einige deckungsgleiche Analyse- und Gestaltungsmethoden zum Service Engineering eingefügt (Prozessanalyse, SWOT-Analyse, From Service to Value, Design Thinking).

Die begleitende Diskussion unter den Teilnehmenden des Expert:innenworkshops lieferte wichtige Einsichten und Hinweise für die weiteren methodenfokussierten Arbeiten im Projekt SO-SERVE. So wurde festgestellt, dass die Gestaltungsmethoden aus dem Service Engineering in der Regel auf technische/digitale Lösungen zur (Weiter-) Entwicklung von DL fokussieren. In der Arbeitswissenschaft liegt der Gestaltungsfokus auf dem Arbeitsinhalt und den Ausführungsbedingungen. Zukünftige Gestaltungsmethoden sollten beide Ansätze integrieren.

Die Teilnehmenden verwiesen auf den Einfluss der COVID-19-Pandemie, die den Einsatz digitaler Werkzeuge in allen Arbeitsbereichen stark vorantreibt. Der Bedarf an einem beschäftigten-orientierten, partizipativ gestalteten Einführungsprozess wurde betont, nicht nur in Bezug auf Digitalisierungsvorhaben. 
Vielen Methoden - sowohl aus der Arbeitswissenschaft als auch aus dem Service Engineering - ist die partizipative Vorgehensweise nach Aussage der Teilnehmenden inhärent. Ebenso bedeutsam ist es, den situativen Kontext von Organisationen bei der Analyse und Gestaltung der Interaktionsarbeit zu berücksichtigen. Als herausfordernd für die menschengerechte Arbeitsgestaltung wurde benannt, dass (digitale) Gestaltungslösungen nur dann zu einer Entlastung von Beschäftigten führen können, wenn neu frei gewordene zeitliche Interaktionsspielräume nicht sofort mit neuen Anforderungen gefüllt werden. Es wurde darauf verwiesen, dass diesbezüglich ein Umdenken erforderlich ist, der Wert von Interaktion anerkannt/wertgeschätzt und zeitlicher Spielraum für situativ angepasstes Arbeitshandeln geschaffen werden muss. 


\section{Methodenzusammenstellung für Interaktionsarbeit}

Im Folgenden soll eine Auswahl von Methoden vorgestellt werden, welche sich für die Gestaltung von Interaktionsarbeit eignen. Die Zusammenstellung enthält sowohl Methoden aus der Arbeitswissenschaft als auch aus dem Service Engineering und gliedert sich in Analyse-, Entwicklungs- und Implementierungsmethoden.
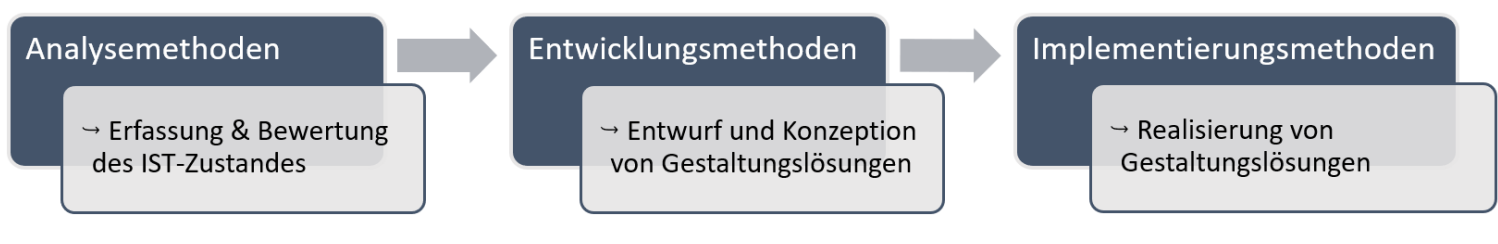

Auswahl von Methoden für die Erfassung und Bewertung des IST-Zustandes

\begin{tabular}{|c|c|}
\hline $\begin{array}{l}\text { Analyse- } \\
\text { methode }\end{array}$ & Beschreibung \\
\hline $\begin{array}{l}\text { Arbeitsan- } \\
\text { forde- } \\
\text { rungserfas- } \\
\text { sung }\end{array}$ & $\begin{array}{l}\text { Ziel der Erfassung der Arbeitsanforderungen ist es, die } \\
\text { vorliegenden Arbeitsbedingungen systematisch und } \\
\text { möglichst objektiv zu erheben. Einen Überblick über re- } \\
\text { levante Analysebereiche geben Listen, wie z. B. die } \\
\text { Merkmalsbereiche der Gefährdungsbeurteilung nach § } \\
5 \text { ArbSchG oder nach der Leitung des Arbeitspro- } \\
\text { gramms Psyche der Gemeinsamen Deutschen Arbeits- } \\
\text { schutzstrategie (GDA, 2017). } \\
\text { Für die konkrete Arbeitsanforderungserfassung bei In- } \\
\text { teraktionsarbeit können als Methoden bspw. die Be- } \\
\text { obachtung, das Beobachtungsinterview sowie die } \\
\text { schriftliche oder mündliche Befragung eingesetzt wer- } \\
\text { den. }\end{array}$ \\
\hline
\end{tabular}

Arbeitsbe- Bei der Arbeitsbewertung werden die, im Rahmen der wertung Arbeitsanforderungserfassung dokumentierten, Arbeitsanforderungen mit einem Bewertungsmaßstab abgeglichen. Als Bewertungsmaßstab für die menschengerechte Arbeitsgestaltung können z. B. ISO-Normen (DIN EN ISO 9241-219: 2019; EN ISO 614-2:2000; DIN EN ISO 6385:2016) herangezogen werden.

Referenz

$\S 5 \mathrm{Arb}-$

SchG;

GDA

(2017)

DIN EN ISO

9241-219:

2019;

DIN EN ISO

614-2:

2008;

DIN EN ISO

Humankriterien sind Merkmale der menschengerech- 6385: 2016 ten Arbeitsgestaltung. Sie umfassen unter anderem Vollständigkeit/ Ganzheitlichkeit der Arbeitseinheiten, Bedeutsamkeit, Anforderungsvielfalt, Handlungsspiel- 
raum, Rückmeldungen, Qualifikationsnutzung und Lernen, Vermeidung von Über- und Unterforderung und Vermeidung sozial isolierender Arbeit.

Beobach- Mittels Beobachtung können in der Interaktionsarbeit tung Abläufe, Ereignisse oder Bedingungen strukturiert erfasst werden (z. B. Beobachtung des Prozesses der Patient:innenversorgung). Die Daten-erhebung erfolgt durch geplante, zielgerichtete und aufmerksame Wahrnehmung. Entsprechend des Beobachtungsziels werden die Beobachtungsinhalte beschrieben und klassifiziert. Durch den Einsatz fachkundiger und geschulter Beobachter:innen können Beobachtungs-ergebnisse objektiver sein als Befragungsergebnisse.

Beobach- Das Beobachtungsinterview ist eine Methode der Artungsinter- beitsanalyse, welche die Methoden Beobachtung und view Interview (mündliche Befragung kombiniert. Das Beobachtungsinterview wird eingesetzt, um Informationen über die Arbeitsbedingungen (Arbeitsinhalt und Ausführungsbedingungen) zu erhalten.

Befragung Die Befragung ist eine Datenerhebungsmethode, die mündlich oder schriftlich durchgeführt werden kann. Eine mündliche Befragung findet in Form eines Interviews bzw. Gesprächs zwischen einem oder mehreren Interviewern auf der einen und einem oder mehreren Interviewten auf der anderen Seite statt. Bei einer schriftlichen Befragung erfolgt die Datenerhebung mittels Fragebogen.

Critical In- Ein kritisches Ereignis (critical incident) ist ein Schlüsselcident ereignis, welches von Kund:innen als besonders (positiv Technik oder negativ) bewertet wird. Bei der Critical Incident Technik werden am DL-Prozess beteiligte Stakeholder im Rahmen eines Interviews dazu aufgefordert über Kuhlmann (2009)

Döring und Bortz (2016) diese kritischen Ereignisse möglichst umfassend zu berichten (Beschreibung von Zeitpunkt, Ort, Reaktion der dienstleistenden Person, etc.), um dadurch besonders effektives bzw. ineffektives Arbeitsverhalten der dienstleistenden Person identifizieren zu können.

Flanagan (1954); Gelbrich (2007) 


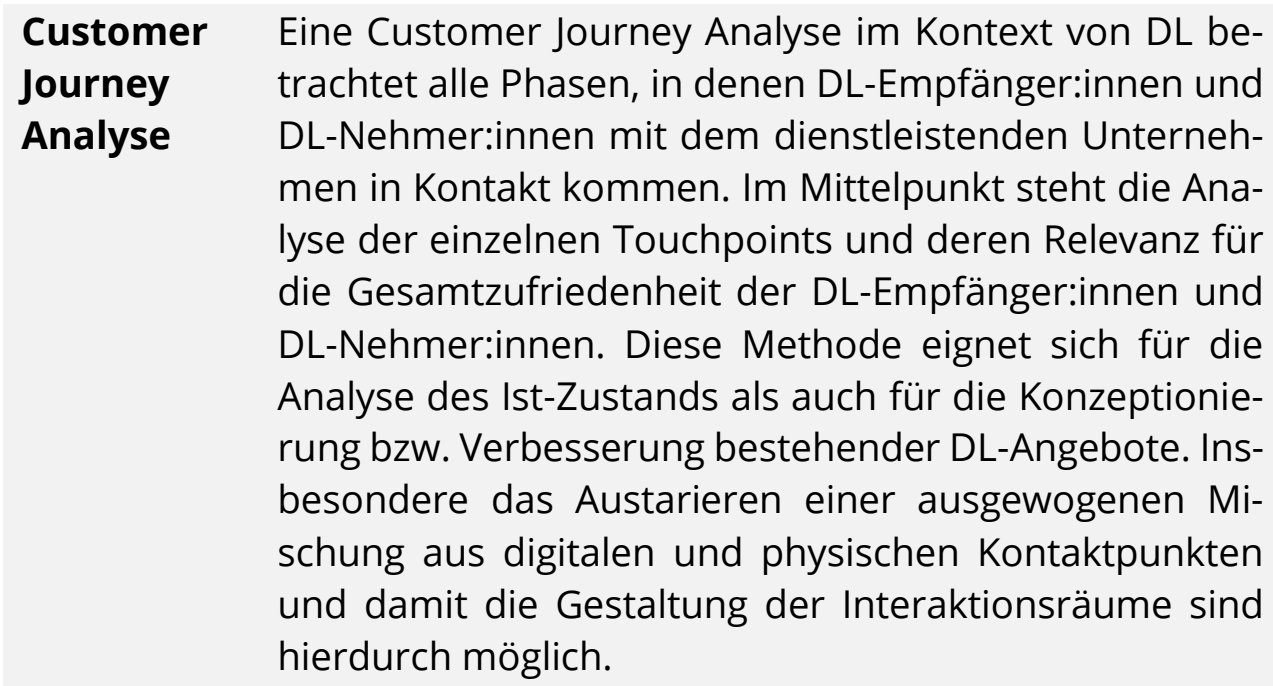

Dokumen- Mittels Dokumentenanalyse werden Informationen tenanalyse über die Arbeit an und mit Menschen aus vorliegenden Dokumenten entnommen und inhaltsanalytisch (z. B. Mayring, 2015) ausgewertet. Als Dokumente für die Analyse dienen bspw. Stellenbeschreibungen, Tarifverträge, Arbeitsanweisungen, Tätigkeitsdokumentationen oder Informationsmaterialien für DL-Nehmer:innen. So lassen sich u. a. Informationen über die Rahmenbedingungen von Interaktionsarbeit, über Arbeitsaufgaben, Arbeitszeitmodelle oder Qualifizierungsangebote erheben.

GAP-Ana- Die Methode der GAP-Analyse dient der Ermittlung von lyse Gründen für eine niedrige Zufriedenheit bei DL-Nehmer:innen, welche als Ergebnis aus Erwartungshaltung und wahrgenommener DL-Qualität angesehen wird. Die Gaps bzw. Unstimmigkeiten deuten dabei auf die Ursachen für die ggf. niedrige Qualitätswahrnehmung durch die DL-Nehmer:innen hin und helfen dem DL-Unternehmen somit, deren Bedürfnisse besser zu verstehen.

Gesund- Gesundheitszirkel sind regelmäßige Workshops, die mit heitszirkel dem Ziel durchgeführt werden, die betriebliche Gesundheitsförderung zu verbessern. Aufgabe innerhalb der Gesundheitszirkel ist es, arbeitsbedingte Gesundheitsgefahren aufzuspüren, zu analysieren und konkrete Vorschläge zur Verbesserung der Arbeitsbedingungen zu erarbeiten. Ein Gesundheitszirkel setzt sich in der Regel aus mehreren Beschäftigten, der Fachkraft für Arbeitssicherheit, dem Betriebsarzt, einem Mitglied des
Bruhn und Hadwich

(2012)

Mayring

(2015)

Schneider et al. (2006)

Slesina und Broekman $n$ (1992) 
Betriebsrats und einem Vertreter des Arbeitgebers zusammen.

Gefähr- Eine Gefährdungsbeurteilung ist ein systematischer dungsbeur- Prozess der Ermittlung und Bewertung relevanter arteilung beitsbedingter Gefährdungen, auf deren Basis erforderliche Maßnahmen festgelegt und umgesetzt werden.

$\S 5$ ArbSchG; GDA (2017) Die Durchführung und Dokumentation der Gefährdungsbeurteilung ist gemäß § 5 Arbeitsschutzgesetz für Arbeitgeber:innen verpflichtend. Die Gefährdungsbeurteilung dient der Sicherheit und dem Gesundheitsschutz der Beschäftigten bei der Arbeit. Gefährdungen können sich u. a. durch die Einrichtung der Arbeitsstätte bzw. des Arbeitsplatzes, die Arbeitsmittel sowie physikalische, chemische oder biologische Einwirkungen ergeben, aber auch durch psychische Belastungen bei der Arbeit.

Pro- $\quad$ Die Ist- und Sollzustandsanalyse der Prozesse erfolgt zessanalyse und vor dem Hintergrund der DIN EN ISO 9000 bzw. 9001. In einem (Geschäfts-)Prozessmodell werden die für die -modellie- Leistungserbringung notwendigen Aktivitäten bzw. Arrung

Service beitsschritte in ihrer zeitlich-sachlichen Abfolge aufgeführt und in Abhängigkeit zueinander gestellt. Die Prozessmodellierung ist somit eine Methode zur systematischen Erstellung von Geschäftsprozessmodellen. Die objektive Darstellung der Prozesse schafft die Grundlage für Optimierungen.

Zum Einsatz kommen Werkzeuge wie PDCA Zirkel, Ishikawa Modell und BPMN-Tools.

Blueprinting

Beim Service Blueprint handelt es sich um eine Form der DL-Modellierung, die sowohl zu Analyse- als auch Gestaltungszwecken angewandt werden kann. Dabei werden einzelne Aktivitäten eines DL-Prozesses in ihrer zeitlichen Reihenfolge geordnet dargestellt, und in (für Kund:innen) sichtbare und unsichtbare Prozesse durch eine "Line of Visibility" getrennt. Im Rahmen der Modellierung wird weder auf die Dienstleitungsumgebung, noch auf Input- oder Outputfaktoren eingegangen.

SWOT-Ana- Die Analyse und Beurteilung der Stärken, Schwächen, lyse Möglichkeiten und Potenziale von Organisationen ist eine weit verbreitete Methode, die internen Prozesse und das Verhältnis zur Umgebung der Organisation zu beurteilen und neue Entwicklungswege abzuleiten.
DIN EN ISO

9000;

DIN EN ISO

9001;

DIN ISO

50001

Gelbrich

(2007)

Gürel

(2017) 


\section{Auswahl von Methoden für den Entwurf und die Konzeption von Gestal- tungslösungen}

\begin{tabular}{|c|c|}
\hline $\begin{array}{l}\text { Gestal- } \\
\text { tungs- } \\
\text { methode }\end{array}$ & Beschreibung \\
\hline $\begin{array}{l}\text { Aufgaben- } \\
\text { bezogener } \\
\text { Informati- } \\
\text { onsaus- } \\
\text { tausch }\end{array}$ & $\begin{array}{l}\text { Der Aufgabenbezogene Informationsaustausch hat zum } \\
\text { Ziel, partizipativ komplexe Probleme, die aus den Ar- } \\
\text { beitsinhalten, der Arbeitsorganisation, und/oder den Ar- } \\
\text { beitsumgebungsbedingungen resultieren, zu lösen. Der } \\
\text { Aufgabenbezogene Informationsaustausch basiert auf } \\
\text { dem Konzept der temporären Kleingruppenarbeit (z. B. } \\
\text { Leitungsgruppen oder Pflegeteams als Kleingruppen). } \\
\text { Es geht beim Aufgabenbezogenen Informationsaus- } \\
\text { tausch um (1) ein gemeinsames Ziel, nämlich die Lösung } \\
\text { des Problems, (2) das Identifizieren der Ursachen von } \\
\text { Problemen sowie um (3) die Sammlung und den Aus- } \\
\text { tausch von Informationen, (4) die Entwicklung von ge- } \\
\text { meinsamen Ideen und Lösungsvorschlägen sowie (5) de- } \\
\text { ren Umsetzung. }\end{array}$ \\
\hline $\begin{array}{l}\text { Business- } \\
\text { Model-Can- } \\
\text { vas }\end{array}$ & $\begin{array}{l}\text { Anhand verschiedener Kriterien wird mit den Beteiligten } \\
\text { aus dem Unternehmen darüber beraten, wie das eigene } \\
\text { Geschäftsmodell und damit Produkte und } D L \text {, mitsamt } \\
\text { derer Kontexte, besser auf die Wünsche und Bedürfnisse } \\
\text { der Kund:innen abgestimmt werden kann. }\end{array}$ \\
\hline $\begin{array}{l}\text { Brainstor- } \\
\text { ming }\end{array}$ & $\begin{array}{l}\text { Brainstorming ist eine Kreativitätstechnik, die die Erzeu- } \\
\text { gung von neuen, ungewöhnlichen Ideen in einer Gruppe } \\
\text { fördern soll. Während der Ideenfindungsphase werden } \\
\text { die Teilnehmenden aufgefordert, ohne jede Einschrän- } \\
\text { kung Ideen zu produzieren. Wertungen und Kritik der } \\
\text { Beiträge, Ideen und Lösungsvorschläge sind nicht er- } \\
\text { laubt. Erst in der Ideenbewertungsphase werden die Er- } \\
\text { gebnisse sortiert und bewertet. Varianten des Brainstor- } \\
\text { mings sind das Brainwriting (schriftliche Ideensamm- } \\
\text { lung) und das Online-Brainstorming (mittels elektroni- } \\
\text { scher Meetingsysteme können Einflüsse der Gruppendy- } \\
\text { namik durch Anonymisierung und Parallelisierung der } \\
\text { Eingaben ausgeschaltet werden). }\end{array}$ \\
\hline
\end{tabular}

Referenz

Pietzcker

und Looks

(2010)

Murray

und

Scuotto

(2015)

Dennis

und

Valacich

(1993) 
Customer Experience Design

Empathy map

From Service to Value

Game Thinking

Handlungsleitfaden
Das Kund:innenerlebnis umfasst die Summe der Erfahrungen und Erlebnisse von Kund:innen bzw. DL-Empfänger:innen mit einem DL-Anbieter. Bei der Gestaltung einer Customer Experience geht es darum, ein möglichst umfassend positives Kund:innenerlebnis (Erlebniskette) zu kreieren, indem förderliche Rahmenbedingungen für die im Zuge der DL stattfindenden Tätigkeiten geschaffen werden.

Um ein detalliertes Profil der Kund:innen und deren Perspektive zu erstellen, bietet sich die Empathy-Map-Methode an. Dafür werden die Denkweisen, Werte, Erfahrungen, Gefühle, Wünsche usw. bestimmter oder fiktiver Kund:innen festgehalten und können so (wie die Persona-Beschreibung, s.u.) in die Gestaltung der DL einfließen. Die Empathy Map (bzw. das Empathie-Raster) kann als eine Ergänzung der Persona-Beschreibung dienen.

Mithilfe dieser Methode wird analysiert, welche Werte (Value in Use) Akteur:innen potenziell aus einer DL ziehen. Sie unterstützt Unternehmen dabei, ihre DL anzupassen und dadurch besser gestalten zu können. Die Methode dient dazu, DL-Angebote ebenso wie die erhaltene Leistung daraufhin zu prüfen, ob und wie sie einen Wert für die jeweiligen Akteur:innen darstellen kann.

Beim Game Thinking handelt es sich um einen Ansatz zur Gestaltung von Lösungen. Indem Probleme wie beim Spieldesign angegangen werden, soll bereits in der Designphase die Motivation und das Nutzenerlebnis der späteren Nutzer:innen bedacht werden.

Ein Leitfaden ist eine „kurz gefasste Darstellung zur Einführung in ein Wissensgebiet" (Duden, 2021). Ein Handlungsleitfaden ist eine möglichst knappe schriftliche Beschreibung zur Ausführung bestimmter Vorgänge oder zum Umgang mit bestimmten Situationen, die Personen (z. B. DL-Geber:innen und DL-Empfänger:innen) darin unterstützt, Vorgänge sachgerecht auszuführen oder mit bestimmten Situationen kompetent umzugehen. Handlungsleitfäden beziehen sich auf einen begrenzten Themenbereich, für den sie Orientierung ermöglichen und konkrete Handlungsoptionen aufzeigen (z. B. Umgang mit Beschwerden, Verhalten in Gefährdungssituationen). Handlungsleitfäden werden für bestimmte The-
Teixeira et

al. (2012)

Cairns et

al. (2021)

Robra-

Bissantz et al. (2021)

Werbach und Hunter (2012) Duden (2021) 
men von zuständigen externen Stellen zur Verfügung gestellt, können aber auch unternehmensintern erstellt werden.

Ideenma- Ideenmanagement meint den planvoll gelenkten Pronagement zess der Ideengenerierung, Ideensammlung und AusSchat wahl geeigneter Ideen in Unternehmen, mit dem Ziel der kontinuierlichen Verbesserung. Um das Ideenmanagement zu unterstützen, können bspw. (digitale) Ideenbriefkästen eingerichtet werden, die Rolle einer Ideenmanagerin / eines Ideenmanagers etabliert werden oder KANBAN-Boards zur Visualisierung genutzt werden.

Kleingrup- Unter Kleingruppenarbeit wird eine heterogene Gruppe penarbeit von Methoden verstanden, bei der eine Gruppe von etwa drei bis acht Personen zeitweilig oder dauerhaft einen gemeinsamen Arbeitsauftrag erfüllt bzw. ein gemeinsames Ziel verfolgt. Formen der Kleingruppenarbeit sind bspw. Workshop, Qualitätszirkel / Gesundheitszirkel, Fokusgruppe, Fallgruppe und Aufgabenbezogener Informationsaustausch. In der Arbeitspsychologie wird Kleingruppenarbeit sowohl für die Arbeitsanalyse und bewertung, als auch für das gemeinsame Ableiten und Umsetzen von Arbeitsgestaltungsmaßnahmen eingesetzt.

Kopfstand- Die Kopfstand-Technik ist eine Kreativitäts- oder ProbTechnik lemlösemethode, bei der die Fragestellung ins Gegenteil verkehrt wird. Beispielsweise wird die Frage "Wer ist von negativen Auswirkungen einer Maßnahme betroffen?" umgekehrt in die Frage "Wer profitiert von den Auswirkungen einer Maßnahme?". Durch den "Kopfstand" sollen neue Perspektiven eröffnet und neue Antworten gefunden werden.

Lego ${ }^{\circledR}$ Seri- $\quad$ Mithilfe dieser kreativen Methode erarbeiten Teams inous Play ${ }^{\circledR} \quad$ nerhalb von Workshops auf Basis der vorhandenen Strukturen neue Perspektiven und Ideen, zum Vertrieb ihrer Produkte und DL. Die Annäherung an die im Workshop zu erarbeitende Lösung geschieht über das geHacker (2018b)

Freitag (2020)

Kristiansen und Rasmusse $n(2014)$ meinschaftliche Bauen mit Legosteinen und den sich dadurch ergebenden Austausch.

Lern- und Anhand verschiedener Kriterien wird mit den Beteiligten Experimen- aus dem Unternehmen darüber beraten, wie das eigene Bundesmi nisterium tierraum für Arbeit 
Geschäftsmodell besser auf die Wünsche und Bedürfnisse der Kund:innen abgestimmt werden kann.

und

Soziales

(2021)

Mind-Map- Das Mind-Mapping ist eine Kreativitätstechnik, bei der

Buzan und ping Gedanken verschriftlicht und in grafischen DarstellunBuzan gen festgehalten werden. Die resultierende Darstellung wird als Mind-Map bezeichnet. Das Hauptthema der Mind-Map wird zumeist mittig angeordnet. Davon ausgehend werden Verbindungen zu den Unterthemen gezogen. So können Informationen zu einem Thema in übersichtlicher Form gesammelt, geordnet und miteinander in Beziehung gesetzt werden.

Mockup Mockups sind grafische Darstellungen der Benutzungsoberfläche einer Software. Sie dienen dazu, über notwendige Funktionalitäten einer Anwendung und ihre Darstellung zu reflektieren sowie mit den Erwartungen der späteren Nutzer:innen abzugleichen. Ein Mockup fungiert in diesem Sinne als Diskussionsgrundlage, welche ohne den Programmieraufwand der späteren funktionalen Anwendung erstellbar ist.

Partizipa- Bei partizipativen Methoden der Arbeitsgestaltung wertive Meden die Beschäftigten in die Neugestaltung oder Veränthoden der derung von Arbeitsprozessen und -bedingungen einbeArbeitsge- zogen. Dies hat den Vorteil, dass Beschäftigte - als Exstaltung pert:innen in der Ausführung der eigenen Arbeit - ihre Blickwinkel und ihre Kenntnisse über die Tätigkeit in die Gestaltung einbringen können. Zudem steigert die Partizipation an Veränderungsprozessen die Akzeptanz für nachfolgende Gestaltungsmaßnahmen. Möglichkeiten für die partizipative Arbeitsgestaltung bieten bspw. Formate wie Gesundheitszirkel oder der Aufgabenbezogene Informationsaustausch.

Persona Personae/Personas finden vielfältigen Einsatz, bei der kundschaftsorientierten Produkt- und DL-Entwicklung. DIN SPEC 33453 Hacker (2018b)

Chang et Sie entstammen v. a. den Methoden des Usability Engial. (2008) neering. Dafür werden fiktive Personen-Profile idealtypischer Kund:innen beschrieben. Dies beinhaltet meist die Beschreibung von Charakterzügen, Zielen und kann auch Wünsche an eine bestimmte DL definieren. Durch die explizite, idealtypische Kund:innenorientierung ist eine Anwendung der Methode im Bereich Kund:innenund Interaktionsorientierung möglich. 

fundierter Entscheidungen unterstützen. Es werden die positiven (Pro-) Argumente und die negativen (Kontra-) Argumente einer Lösungsvariante systematisch und zumeist tabellarisch gegenübergestellt.

Eine Erweiterung der Pro-Kontra-Analyse liegt in der Bewertung bzw. Gewichtung der einzelnen Argumente (Nutzwertanalyse). Durch die Gewichtung wird die Bedeutung der jeweiligen Pro- und Kontra-Argumente berücksichtigt.

In dieser Methode wird die DL in einem Rollenspiel nachgespielt, um so die unterschiedlichen Erfahrungen, Funktionen und Touchpoints zu veranschaulichen. So kann etwa die Interaktionssituation in einem solchen Role Play gespielt werden. Durch ein anschließendes Stakeholder Feedback können die einzelnen Perspektiven verdeutlicht und analysiert werden, woraus dann Verbesserungspotenziale für die $\mathrm{DL}$ deutlich werden.

Scenario

Building / Szenariotechnik $\begin{array}{lll}\text { Service- } & \text { In Workshops zum Service-Ökosystem-Design wird der } & \text { Robra- } \\ \text { Ökosys- } & \text { derzeitige Stand des Service-Ökosystems hinsichtlich der } & \text { Bissantz, } \\ \text { tem-Design } & \text { Akteur:innen sowie der Services oder Kompetenzen, die } & \text { Lux, } \\ & \text { sie untereinander austauschen, visualisiert (z. B. in Form } & \text { Lattemann } \\ & \text { von Karten). Für alle Akteur:innen erfolgt anschließend } & 2021 \\ & \text { ein Abgleich der Werte, die sie erhalten, sowie der Kom- } \\ & \text { petenzen, die sie dafür einsetzen (Werte-Kompetenzen- } \\ & \text { Matrix). Stellt sich heraus, dass dem Service-Ökosystem } \\ & \text { wichtige Partner:innen oder Kompetenzen fehlen, so } \\ & \text { wird mithilfe von so genannten Patterns (Best-Practice- } \\ & \text { Mustern) im Service-Ökosystem simuliert, wie es um bei- } \\ & \text { spielsweise neue Akteur:innen oder neue digitale }\end{array}$ van Husen et al. (2020)

Kosow und León (2015) dener Stakeholder, um so eine möglichst breite Perspektive auf die verschiedenen Szenarios festzuhalten. Das Ziel dabei ist, verschiedene Risiken, Unsicherheiten und Hürden aufzudecken, die dem auf dem Weg zum gewünschten Ziel (d. h. eine gelungene Interaktion) liegen können. Dadurch können z. B. Einzelheiten der Ziele konkretisiert und Strategien und Prozesse angepasst werden.

Im Scenario Building werden verschiedene (mögliche) Zukunftsszenarien in mehreren Phasen definiert und (2015) 
Dienste erweitert werden kann. Die Methode dient der Gestaltung eines nachhaltig funktionierenden ServiceÖkosystems.

SMART-Me- Mit der SMART-Methode kann die Definition konkreter thode

Ziele unterstützt werden. SMART ist ein Akronym, das für

Freitag die Kriterien spezifisch, messbar, attraktiv, realistisch und terminiert steht. Spezifisch steht für die eindeutige Zielbeschreibung; mit Messbar wird festgelegt, woran die Zielerreichung gemessen werden kann; Attraktiv beschreibt den positiven Anreiz der Zielerreichung; Realistisch beinhaltet die praktische Umsetzbarkeit des Ziels und mit Terminiert wird festgelegt, bis wann das Ziel erreicht werden soll.

User Story Die Formulierung von User Stories dient der Definition konkreter Anforderungen an ein System und ist somit ein wichtiger Schritt im Designprozess. Sie sind nach Leffingwell (2011) dem immer gleichen Schema formuliert: Wer möchte was und warum? Im Kontext der Gestaltung von Interaktionsarbeit unterstützen User Stories den Perspektivwechseln und tragen dazu bei, Software (Kommunikationstechnologie) bedarfsgerecht für alle Nutzer:innen zu gestalten.

Als [Rolle/Nutzer:in] möchte ich [Funktion/Wunsch], damit/um/weil [Nutzen/Wert].

Value

Das Value Proposition Canvas ist Ansatz zur StrukturieProposition Canvas rung des eigenen Leistungsversprechens (value proposition) im Rahmen der DL-Gestaltung. Das Modell unterscheidet zwei Bereiche: Zum einen enthält es das Kund:innensegment, welches die Kund:innenaufgaben, Probleme (Pains) und Erwartungen (Gains) der Kund:innen auflistet. Zum anderen beinhaltet es eine Darstellung des Leistungsversprechens (value map), in welchem Produkte und DL, Pain Relievers - also Lösungen, die den Zweck erfüllen, negative Kund:innenerfahrungen zu beseitigen - und Gain Creators - also Nutzenpunkte, die sich für Kund:innen durch die Inanspruchnahme der angebotenen DL ergeben - dargestellt werden.

Virtual Re- Die Möglichkeiten der Visualisierung komplexer DL-Sysality teme mithilfe von Virtual Reality bietet eine große Hilfestellung für Entwickler:innen und Entscheider:innen. In Kombination mit Konzepten wie dem Service Theater

Kyhnauund Nielsen (2015)

Meiren und Freitag (2018) 
wird es zudem möglich, gänzlich neue Arten von Prototypen für DL zu erstellen und insbesondere das kundschafts- und beschäftigtenorientierte Testen neuer DL wirkungsvoll zu unterstützen. 


\section{Auswahl von Methoden für die Realisierung von Gestaltungslösungen}

\begin{abstract}
Realisie-
rungsme-

thode

Ergonomi-

sche Ar-

beitsmittel

Beschreibung

Ergonomische Arbeitsmittel sind Arbeitsmittel, die an die Bedürfnisse und Fähigkeiten des arbeitenden Menschen angepasst sind. Unter Arbeitsmitteln versteht man allgemein alle erforderlichen Komponenten für den Arbeitsprozess, die von DL-Geber:innen, ggf. unter Mitwirkung der DL-Empfänger:innen eingesetzt werden, bspw. Verbrauchsmaterial, Mobiliar, Hard- und Software. Der Einsatz ergonomischer Arbeitsmittel soll die körperliche und psychische Beanspruchung optimieren.
\end{abstract}

Referenz

Festlegung Die Festlegung von Erreichbarkeitszeiten dient dazu, die von ErreichbarEinhaltung der Regelungen des Arbeitszeitgesetzes zu unterstützen und Erholungszeiten für Beschäftigte zu keitszeiten gewährleisten. Auch in der Interaktionsarbeit wird zunehmend mit digitalen Arbeitsmitteln gearbeitet, die eine orts- und zeitunabhängige Kommunikation mit verschiedenen Akteur:innen des DL-Netzwerks ermöglichen (in Kitas z. B. Kita-Leitung, Kolleg:innen und Eltern). Damit diese Möglichkeiten nicht zu einer Entgrenzung der Arbeitszeit führen, müssen Erreichbarkeitszeiten für Beschäftigte festgelegt werden, die mit den vertraglich festgelegten Arbeitszeiten im Einklang sind. Die Erreichbarkeitszeiten sollten den Interaktionspartner:innen innerhalb des DL-Netzwerks kommuniziert werden.

Job Rota- Job Rotation (Tätigkeitswechsel) ist eine Methode der Artion beitsgestaltung mit dem Ziel, die Anforderungsvielfalt bei der Arbeit zu erhöhen. Beschäftigte wechseln nach einem systematisch en Rotationsverfahren zwischen verschiedenen Arbeitsplätzen. Im Optimalfall erfolgt die Rotation zwischen qualitativ unterschiedlichen Arbeitsanforderungen, um Qualifikationsnutzung und Lernen zu fördern.

Job Enlar- Job Enlargement (Aufgabenerweiterung) ist eine Megement
DIN EN ISO 6385: 2016; DIN Normenreihe ISO 9241

Pangert et al. (2016)

Ulich

(2005)

Ulich

(2005) thode der Arbeitsgestaltung mit dem Ziel, die Anforderungsvielfalt bei der Arbeit zu erhöhen. Sie besteht darin, mehrere Aufgaben mit qualitativ gleichen oder ähnlichen Arbeitsanforderungen aneinanderzureihen und dadurch den Arbeitszyklus zu erweitern. Im Gegensatz 
zur Aufgabenbereicherung können Qualifikationsnutzung und Lernen durch diese Methode nicht gefördert werden.

Job Enrich- Job Enrichment (Aufgabenbereicherung) ist eine Mement thode der Arbeitsgestaltung mit dem Ziel, die Anforderungsvielfalt einer Tätigkeit zu erhöhen sowie Qualifikationsnutzung und Lernen zu fördern. Bei der Aufgabenbereicherung werden den bestehenden Aufgaben neue qualitativ höherwertige Aufgaben hinzugefügt.

KANBAN- Das KANBAN-Board ist ein Tool zur Unterstützung eines Board strukturierten Ideenmanagements. Es dient der Visualisierung von Arbeitsaufträgen (im Hinblick auf die Umsetzung von Ideen) und Bearbeitungsständen und macht diese transparent für alle am Veränderungsprozess Beteiligten.

Pausenge- Der Begriff Pausengestaltung umfasst eine Vielzahl bestaltung trieblicher Maßnahmen, um die gesetzlich vorgeschriebenen Pausen der Beschäftigten zu gestalten. Dies betrifft u. a. die Pausenlänge (z. B. Kurzpausensystem), das Pausenintervall, den Pausenort, den Pauseninhalt (z. B. aktive Pause) und soziale Aspekte (z. B. Pause gemeinsam mit anderen). Ein Mitspracherecht der Beschäftigten bei der Pausengestaltung kann zu einem stärkeren Erholungseffekt führen.

RACl- Das Aufstellen einer RACl-Matrix dient der Klärung und Matrix Visualisierung von Verantwortlichkeiten und Aufgaben in Teams und kann im Kontext der Implementierung neuer DL dazu genutzt werden, neue Aufgaben oder Aufgabenbereichen verbindlich bestimmten Personen zuzuweisen. RACl ist ein Akronym und bedeutet: R (Responsible: Wer ist für die Durchführung der Aufgabe verantwortlich?), A (Accountable: Wer entscheidet, ob die Aufgabe korrekt durchgeführt wurde?), C (Consulted: Wer wird zur Durchführung der Aufgabe befragt / wer kann unterstützen [z. B. Fachexpert:innen und Dritte]?), I (Informed: Wer wird über die Aufgabendurchführung oder die Arbeitsergebnisse informiert?)

Rollenfest- Die Festlegung von Rollen und Verantwortlichkeiten in legung Teams dient dazu, Verantwortungsdiffusion und rollenBotta bezogene Konflikte zu vermeiden. Das dafür genutzte KusayMerkle (2018)

Wendsche und LohmannHaislah (2018)

Smith und Erwin (2005) Role Model Canvas ist ein Template bzw. ein Plakat, wel(2016) GDA (2017) ches eine Rolle über acht Felder definiert. Dieses wird in

Ulich
(2005) ) 
Form von Workshops von den Teammitgliedern und Vertreter:innen der Leitungsebene ausgefüllt.

Teambuil- Unter Teambuilding versteht man eine größere Gruppe ding an Methoden, die die Zusammenarbeit in Teams verbessern sollen. Ziele sind z. B. die Erhöhung von Teameffizienz oder Stärkung des Zusammengehörig-keitsgefühls. Themen für das Teambuilding werden in Abhängigkeit der Entwicklungsphase des Teams ausgewählt (Forming,

Becker (2016) Tuckman und Jensen (1977) Storming, Norming, Performing, Adjourning; vgl. Tuckman \& Jensen, 1977) und können bspw. das Rollen- und Aufgabenverständnis, Konfliktlösung im Team, das Setzen von Teamzielen oder die Vertrauenskultur betreffen. Prominent sind auch die unter „Teambuilding-Events" bekannten Teamerlebnisse und Betriebsausflüge (z. B. gemeinsamer Floßbau, Sportevent, Team-Übungen).

Teilauto- Die Bildung teilautonomer Arbeitsgruppen ist eine Menome Ar- thode der Arbeitsgestaltung, bei der einer Gruppe von Ulich (2005) beitsgruppen reich zur Erledigung in eigener Verantwortung übertragen wird. Die Mitglieder der teilautonomen Arbeitsgruppe sind in der Regel für einen ganzen Prozess, ein Produkt oder eine DL verantwortlich. Abhängig davon, welche Entscheidungsbefugnisse der Gruppe übertragen werden (vgl. Handlungsspielraum: z. B. eigenständiges Setzen von Zielen bzw. Teilzielen, Arbeitsort, Arbeitszeit, interne Aufgabenverteilung), sind in der Praxis unterschiedliche Grade der Autonomie möglich. 


\section{Prinzipien des Social Service Engineering}

Mit der Formulierung von Prinzipien für Social Service Engineering werden Leitlinien für die Gestaltung von Interaktionsarbeit beschrieben. Diese wurden, unter Hinzunahme von Anregungen aus dem Expert:innen-Workshop, im Rahmen des Projekts SO-SERVE ausgearbeitet.

Übergeordnetes Ziel des Social Service Engineering ist es, Interaktionsarbeit sowohl menschengerecht als auch wirtschaftlich zu gestalten, unter Nutzung von Synergien aus Arbeits- und DL-Gestaltung. Nachhaltiger Unternehmenserfolg ist nur durch eine gesundheits-, lern- und persönlichkeitsförderliche Arbeitsgestaltung möglich, die zugleich an eine wirtschaftliche Unternehmensführung unter Berücksichtigung der Wünsche von DL-Empfänger:innen und DL-Nehmer:innen gekoppelt ist. Daraus leiten sich die folgenden Grundsätze für die Gestaltung menschengerechter und effizienter Interaktionsarbeit ab, welche gleichwertig zu behandeln sind und gleichzeitig eingehalten werden sollen:

Im Social Service Engineering müssen sowohl Aspekte der Arbeitsgestaltung als auch der Dienstleistungsgestaltung gemeinsam betrachtet und gestaltet werden.

Wechselwirkungen zwischen guten Arbeitsbedingungen für DL-Geber:innen und einer hohen DL-Qualität für die DL-Empfängerinnen und DLNehmer:innen beeinflussen die Zufriedenheit auf beiden Seiten und potenzieren damit den Unternehmenserfolg. Aus diesem Grund müssen beide Aspekte bei der Gestaltung der Interaktionsarbeit Berücksichtigung finden. Im Optimalfall entsteht durch die Gestaltung der Interaktionsarbeit eine Win-Win-Situation für alle Beteiligten. 
Der Fokus des Social Service Engineering muss auf der Zeit für das empathisch-mitmenschliche Zuwenden liegen.

Die Interaktion zwischen Menschen stellt den eigentlichen Kern und Wert von Interaktionsarbeit dar. Nur die persönliche, zwischenmenschliche Interaktion und Zuwendung gewährleistet, dass Interaktionsarbeit sowohl den Ansprüchen der DL-Empfänger:innen, DL-Nehmer:innen und des Unternehmens als auch dem professionellen Selbstanspruch der DL-Geber:innen gerecht wird. Für diese Interaktion ist Zeit zu schaffen. Dies kann zum Beispiel erreicht werden, indem die an die Interaktionsarbeit gekoppelten Unterstützungsprozesse, nämlich Organisations, Koordinations- und Beschaffungsprozesse, erleichtert werden, evtl. durch Digitalisierung. Es muss hierbei abgewogen werden, welche Aspekte der DL digitalisiert oder digital unterstützt werden können (z. B. Schichtplanung, reine Informationsbereitstellung) und welche nicht (z. B. lebensberatende Gespräche, Beziehungsgespräche).

Social Service Engineering muss die Voraussetzungen und Möglichkeiten aller Akteur:innen im Netzwerk berücksichtigen.

Sowohl auf der Seite der DL-Geber:innen, als auch auf der Seite der DLEmpfänger:innen und DL-Nehmer:innen sind bei der Gestaltung von Interaktionsarbeit Bedingungen zu berücksichtigen, die das Verhalten und Erleben der Akteure im jeweiligen DL-Kontext beeinflussen. Hierzu gehören $u$. a. organisatorische Voraussetzungen (z. B. zur Verfügung stehende Zeit, technische Ausstattung), emotional-motivationale Voraussetzungen (z. B. Erwartungen an die Interaktion), kognitive Voraussetzungen (z. B. Qualifikation, Kompetenzen im Umgang mit Technik) und soziale Bedingungen (z. B. soziale Normen).

Den Hauptakteur:innen der DL (DL-Geber:innen, DL-Empfänger:innen und DL-Nehmer:innen) müssen sinnvolle Möglichkeiten der Partizipation im Hinblick auf die Gestaltung der Interaktion gegeben werden. Darüber hinaus ist die Partizipation des erweiterten DL-Netzwerkes wünschenswert. 
Service Innovation ist bei Interaktionsarbeit durch die Integration der Akteur:innen in den Innovationsprozess erreichbar. Das Wissen und die Erfahrungen der DL-Geber:innen, DL-Empfänger:innen und DL-Nehmer:innen mit der Interaktion sollten genutzt werden, um herauszufinden, welche Prozesse noch nicht optimal gestaltet sind, und um den Service Innovation Process gemeinsam umzusetzen. Beteiligung dient nicht nur der Nutzung des Expert:innenwissens der von Gestaltungsmaßnahmen Betroffenen, z. B. für die Verbesserung von Arbeitsabläufen oder DL-Prozessen, sondern fördert auch die Wertschätzung der Betroffenen sowie das Überwinden von Widerständen im Veränderungsprozess. Bei der Entwicklung und Einführung von Digitalisierungsmaßnahmen vermeidet das frühzeitige Einbinden späterer Nutzer:innen mögliche Fehlentwicklungen. Zudem werden Möglichkeitsräume werden enorm vergrößert, wenn das gesamte DL-Netzwerk einbezogen wird. So können etwa externe Stakeholder im Sinne eines kollaborativen Netzwerkes in digital gestützte Kommunikationsprozesse eingebunden werden und sich aktiv am Innovationsprozess beteiligen (Open Space Innovations). Ein systematisches Ideen- und Innovationsmanagement ist aufgrund dieser Komplexität zu empfehlen.

\section{Ziele der Organisation müssen klar definiert und aufeinander abge- stimmt sein (Zielkongruenz).}

Klar umschriebene Ziele unterstützen den Gestaltungsprozess, indem sie die Priorisierung und Auswahl von Gestaltungsmaßnahmen erleichtern (Bewertungs- und Selektionsfunktion), die Koordination von betrieblichen Akteur:innen auf gemeinsame Ziele hin fördern (Motivationsfunktion) und die Überprüfung von Zwischenergebnissen in Bezug auf die angestrebten Haupt- und Unterziele ermöglichen (Steuerungsfunktion). Die Ziele sollten im Hinblick auf strategische Unternehmensziele und untereinander stimmig sein, damit sie sich im besten Fall gegenseitig fördern und die Maßnahmen einander nicht entgegenlaufen. 


\section{Gestaltungsmaßnahmen sollen Probleme an ihrer Quelle beheben.}

Gestaltungsmaßnahmen können prinzipiell an zwei Stellen ansetzen: Bedingungsbezogene Maßnahmen zielen auf eine Verbesserung der Bedingungen der DL (z. B. Optimierung von Arbeitsabläufen, Verbesserung der Nutzungsfreundlichkeit eines Software-Produkts). Personenbezogene Maßnahmen bezwecken einen verbesserten Umgang mit den gegebenen Bedingungen (z. B. Erlernen des Umgangs mit Zeitknappheit, Schulung zur Nutzung einer Software).

Werden in Organisationen Gestaltungsbedarfe erkannt, soll bei der Auswahl von Gestaltungsmaßnahmen die Grundregel "bedingungsbezogene Maßnahmen vor personenbezogenen Maßnahmen" beachtet werden (vgl. auch § 4 Satz 2 und 5 ArbSchG, vgl. TOP-Prinzip). Erst, wenn technische oder organisatorische Maßnahmen ausgeschöpft oder nicht geeignet sind, das Problem zu beheben, sollen personenbezogene Maßnahmen eingesetzt werden.

Die Gestaltung von Interaktionsarbeit bedarf einer gemeinsamen Wertebasis, die auf Vertrauen und Transparenz baut und an der sich die Zusammenarbeit im Gestaltungsprozess orientieren kann.

Eine vertrauensbasierte Kollaboration, in der alle Beteiligten konstruktiv mitwirken können, bildet die Grundlage der Gestaltung von Interaktionsarbeit. Diese spiegelt sich in transparenten Gestaltungsprozessen und horizontalen Aushandlungsprozessen wider, in denen sich alle Beteiligten gleichberechtigt einbringen können und möchten. Dies setzt auch neue Führungsansätze voraus, in denen hierarchische Kontrolle und Top-down-Ansätze höchstens minimal eingesetzt werden sollten.

Interaktionsarbeit, insbesondere, wenn sie durch Teams ausgeführt wird, bedarf eines gemeinsamen Service Mindsets.

Das Service Mindset spiegelt die Grundhaltung eines DL-Unternehmens im Hinblick auf die DL-Erbringung wider und schafft eine gemeinsame Basis für gelungene Interaktionsarbeit. Der Service Mindset setzt eine Art Codex mit welchem den Stakeholdern des Wertschöpfungsnetzwerkes gegenübergetreten wird und schafft Handlungssicherheit in Alltags- und 
Krisensituationen. Dabei geht es sowohl um die Ziele, die mit der Erbringung der DL angestrebt werden als auch um die Art und Weise, wie diese Ziele erreicht werden. Neben Wertvorstellungen ist deshalb auch die Rolle der Akteur:innen im Wertschöpfungsprozess Gegenstand des Service Mindsets.

Das Service Mindset sollte partizipativ mit den Beschäftigten des DL-Unternehmens entwickelt werden. Dies stellt sicher, dass das Mindset von den DL-Geber:innen mitgetragen und bei der täglichen Arbeit umgesetzt werden kann. Zudem wird durch die partizipative Entwicklung für die DLGeber:innen leichter nachvollziehbar, warum bestimmte Tätigkeiten in einer bestimmten Weise ausgeführt und bestimmte Methoden angewandt werden. 


\section{Ausblick für die Gestaltung von Interaktionsarbeit}

Der gegenwärtige Transformationsprozess der Arbeit (z. B. Digitalisierung, Flexibilisierung) macht bei der Interaktionsarbeit nicht halt. In der Gestaltung dieses Prozesses ist es wichtig, sich auf das Kernelement der Interaktionsarbeit zurückzubesinnen: die Interaktion zwischen Menschen. In diesem Kern nehmen die Menschen, mit all ihrer Diversität, eine zentrale Rolle ein. Aus diesem Grund muss sich jeder Transformations- und Gestaltungsprozess der Interaktionsarbeit daran messen lassen, welche Werte für die Akteure der Interaktionsarbeit entstehen - das beinhaltet sowohl die Werte für die DL-Empfänger:innen als auch für die DL-Geber:innen. Dieser Gedanke führt zwangsläufig dazu, sich den wissenschaftlichen Disziplinen zu widmen, welche sich mit den Blickwinkeln der DL-Nehmer:innen und der DL-Geber:innen auseinandersetzen. Diese Arbeit setzt hierfür einen Startpunkt. Eine interdisziplinäre Herangehensweise, in der sich Arbeitswissenschaft und Service Engineering gegenseitig ergänzen und so Synergien schaffen, mit denen ein neuer Blick auf Interaktionsarbeit ermöglicht wird, ist hierbei folgerichtig.

Um die Zusammenarbeit und den Austausch über den Gegenstand „Interaktionsarbeit" zu erleichtern, ist der nächste logische Schritt die Entwicklung eines Thesaurus, um ein gemeinsames Verständnis und Vokabular für die interdisziplinäre Arbeit aufzubauen.

Jedoch ist nicht nur ein ständiger Austausch der Wissenschaftszweige wichtig, sondern auch eine enge Zusammenarbeit mit Praxispartner:innen. Die praxisorientierte (Weiter-)Entwicklung von passgenauen Gestaltungsmethoden und Werkzeugen für Interaktionsarbeit muss weiter vorangetrieben werden. Das vorliegende Whitepaper begreift sich als einen ersten Schritt in diese Richtung 
- durch die Definition von Prinzipien und eine erste Sammlung von Methoden ist eine wichtige Grundlage für Wissenschaft und Praxis geschaffen und ein wichtiger Baustein für den Transfer gelegt.

Darüber hinaus ist seitens der Autor:innen ein digital nutzbarer Social Service Engineering Methodenbaukasten und eine Schritt-für-Schritt-Anleitung (Vorgehensmodell) in Entwicklung, mit denen Praktiker:innen bei der eigenständigen Gestaltung der Interaktionsarbeit entsprechend der Social Service Engineering-Prinzipien unterstützt werden. 


\section{Literaturverzeichnis}

Adam, T. (2012). Die Bewertung von Innovationsideen: Eine empirische Analyse von Bewertungsdimensionenund sozialen Einflussfaktoren [Dissertation]. RWTH, Aachen. https://d-nb.info/1023940841/34

Baines, T. S., Lightfoot, H. W., Evans, S., Neely, A., Greenough, R., Peppard, J., Roy, R., Shehab, E., Braganza, A., Tiwari, A., Alcock, J. R., Angus, J. P., Bastl, M., Cousens, A., Irving, P., Johnson, M., Kingston, J., Lockett, $H_{\text {., }}$ Martinez, V., Michele, P., Tranfield, D., Walton, I. M., Wilson, H. (2007). State-ofthe-art in product-service systems. Proceedings of the Institution of Mechanical Engineers, Part B: Journal of Engineering Manufacture, 221(10), 1543-1552. https://doi.org/10.1243/09544054JEM858

Baumgärtner, M. \& Bienzeisler, B. (2006). Dienstleistungsproduktivität: Konzeptionelle Grundlagen am Beispiel interaktiver Dienstleistungen. Fraunhofer IRB Verlag.

Becker, F. (2016). Teamarbeit, Teampsychologie, Teamentwicklung: So führen Sie Teams! (1. Auflage). Springer.

Bleses, P., Busse, B., Friemer, A., Kludig, R., Breuer, J., Philippi, L., Bidmon-Berezinski, J. \& Schnäpp, M. (2018). Verbundprojekt KOLEGE - Interagieren, koordinieren und lernen: Chancen und Herausforderungen der Digitalisierung in der ambulanten Pflege. Zwischenbericht - Ergebnisse der Analysephase (Schriftenreihe Institut Arbeit und Wirtschaft 24/2018). Institut Arbeit und Wirtschaft (IAW), Universität Bremen und Arbeitnehmerkammer Bremen.

Böhle, F., Stöger, U. \& Weihrich, M. (2015). Interaktionsarbeit gestalten: Vorschläge und Perspektiven für humane Dienstleistungsarbeit. Forschung aus der Hans-Böckler-Stiftung: Bd. 168. Edition Sigma.

Böhle, F. \& Weihrich, M. (2020). Das Konzept der Interaktionsarbeit. Zeitschrift für Arbeitswissenschaft, 74(1), 9-22. https://doi.org/10.1007/s41449-02000190-2

Botta, C. (2016). Das Role Model Canvas - Rollen schnell und gemeinsam definieren: Visuelle Alternative zur RACI-Matrix. Berleb Media GmbH.

Böttcher, M. (2009). Architektur integrierter Dienstleistungssysteme: Konzeption, Metamodell und technikraumspezifische Konkretisierung [Dissertation]. Universität Leipzig. 
Bruhn, M. (2020). Qualitätsmanagement für Dienstleistungen. Springer Berlin Heidelberg. https://doi.org/10.1007/978-3-662-62120-2

Bruhn, M. \& Hadwich, K. (2012). Customer Experience - Eine Einführung in die theoretischen und praktischen Problemstellungen. In M. Bruhn \& K. Hadwich (Hrsg.), Customer Experience: Forum Dienstleistungsmanagement (S. 3-36). Gabler Verlag.

Bullinger, H.-J., Fähnrich, K.-P. \& Meiren, T. (2003). Service engineering-methodical development of new service products. International Journal of Production Economics, 85(3), 275-287. https://doi.org/10.1016/S09255273(03)00116-6

Bullinger, H.-J. \& Schreiner, P. (2006). Service Engineering: Ein Rahmenkonzept für die systematische Entwicklung von Dienstleistungen. In H.-J. Bullinger, A.W. Scheer \& O. Grieble (Hrsg.), Service Engineering: Entwicklung und Gestaltung innovativer Dienstleistungen (S. 51-82). Springer. https://doi.org/10.1007/9783-662-09871-4_3

Bundesministerium für Arbeit und Soziales. (2021). Experimentierräume.de. https://www.experimentierraeume.de/start/ (zuletzt geprüft am: 25.05.2021)

Buzan, T. \& Buzan, B. (1993). The mind map book (1. Aufl.). BBC Books.

Cairns, P., Pinker, I., Ward, A., Watson, E. \& Laidlaw, A. (2021). Empathy maps in communication skills training. The clinical teacher, 18(2), 142-146. https://doi.org/10.1111/tct.13270

Chang, Y., Lim, Y. \& Stolterman, E. (2008). Personas: from theory to practices. In NordiCHI 2008 Building Bridges: Proceedings of the 5th Nordic conference on Human-computer interaction building bridges (S. 439-442). ACM.

Dennis, A. R. \& Valacich, J. S. (1993). Computer brainstorms: More heads are better than one. Journal of Applied Psychology, 78(4), 531-537. https://doi.org/10.1037/0021-9010.78.4.531

DIN EN 614-2:2008-12, Sicherheit von Maschinen - Ergonomische Gestaltungsgrundsätze - Teil 2: Wechselwirkungen zwischen der Gestaltung von Maschinen und den Arbeitsaufgaben; Deutsche Fassung EN 614-2:2000+A1:2008. 
DIN EN ISO 10075-2:2000-06, Ergonomische Grundlagen bezüglich psychischer Arbeitsbelastung - Teil 2: Gestaltungsgrundsätze (ISO 10075-2:1996); Deutsche Fassung EN ISO 10075-2:2000.

DIN EN ISO 50001:2018-12, Energiemanagementsysteme - Anforderungen mit Anleitung zur Anwendung (ISO 50001:2018); Deutsche Fassung EN ISO 50001:2018.

DIN EN ISO 6385:2016-12, Grundsätze der Ergonomie für die Gestaltung von Arbeitssystemen (ISO 6385:2016); Deutsche Fassung EN ISO 6385:2016.

DIN EN ISO 9000:2015-11, Qualitätsmanagementsysteme - Grundlagen und Begriffe (ISO 9000:2015); Deutsche und Englische Fassung EN ISO 9000:2015.

DIN EN ISO 9001:2015-11, Qualitätsmanagementsysteme - Anforderungen (ISO 9001:2015); Deutsche und Englische Fassung EN ISO 9001:2015.

DIN EN ISO 9241 (Normenreihe), Ergonomie der Mensch-System-Interaktion.

DIN EN ISO 9241-210:2019-05, Ergonomie der Mensch-System-Interaktion Teil 210: Prozess zur Gestaltung gebrauchstauglicher interaktiver Systeme (ISO/FDIS_9241-210:2019); Deutsche und Englische Fassung EN ISO 9241210:2019.

DIN SPEC 33453:2019-09: Entwicklung digitaler Dienstleistungssysteme.

DIN-Fachbericht 75:1998, Service Engineering - Entwicklungsbegleitende Normung (EBN) für Dienstleistungen.

Döring, N. \& Bortz, J. (2016). Forschungsmethoden und Evaluation in den Sozial- und Humanwissenschaften (5. Aufl.). Springer-Lehrbuch. Springer.

Duden. (2021). Leitfaden, der. https://www.duden.de/node/88957/revision/88993 (zuletzt geprüft am: 21.04.2021)

Dunkel, W. \& Weihrich, M. (Hrsg.). (2012). Interaktive Arbeit. Springer Fachmedien Wiesbaden. https://doi.org/10.1007/978-3-531-19359-5

Ernst, G. \& Zühlke-Robinet, K. (2018). Dienstleistungen - Wissenschaft und Forschung, Arbeit und Innovation (1. Aufl.). Reihe Dienstleistungsmanagement, Dienstleistungsmarketing: Band 4. Nomos.

https://doi.org/10.5771/9783845292052 
Fähnrich, K.-P. \& Opitz, M. (2006). Service Engineering - Entwicklungspfad und Bild einer jungen Disziplin. In H.-J. Bullinger, A.-W. Scheer \& O. Grieble (Hrsg.), Service Engineering: Entwicklung und Gestaltung innovativer Dienstleistungen (S. 85-112). Springer. https://doi.org/10.1007/978-3-662-09871-4_4

Flanagan, J. C. (1954). The critical incident technique. Psychological bulletin, 51(4), 327-358. https://doi.org/10.1037/h0061470

Freitag, E. (2020). Kreativitätstechniken: So finden Sie das richtige Werkzeug für Ihr Problem. expert verlag.

Freund, L. E. \& Spohrer, J. C. (2013). The human side of service engineering. Human Factors and Ergonomics in Manufacturing \& Service Industries, 23(1), 2-10. https://doi.org/10.1002/hfm.20521

GDA. (22. November 2017). Arbeitsschutz in der Praxis: Empfehlungen zur Umsetzung der Gefährdungsbeurteilung psychischer Belastung. Bundesministerium für Arbeit und Soziales. https://www.gda-psyche.de/SharedDocs/Downloads/DE/empfehlungen-zur-umsetzung-der-gefaehrdungsbeurteilung-psychischer-belastung.pdf?_blob=publicationFile (zuletzt geprüft am: 28.05.2021)

Gelbrich, K. (2007). Blueprinting, sequentielle Ereignismethode und Critical Incident Technique. In R. Buber \& H. H. Holzmüller (Hrsg.), Qualitative Marktforschung (S. 617-633). Gabler. https://doi.org/10.1007/978-3-8349-9258-1_39

GfA (Hrsg.). (1973). Denkschrift „Arbeitswissenschaft in der Gesetzgebung". Frankfurt am Main.

GfA. (2016). Selbstverständnis der Gesellschaft für Arbeitswissenschaft e.V. (GfA). http://www.gesellschaft-fuer-arbeitswissenschaft.de/inhalt/dokumente/gfaselbstverstaendnis-2016.pdf (zuletzt geprüft am: 28.05.2021)

Gremyr, I., Valtakoski, A. \& Witell, L. (2019). Two routes of service modularization: advancing standardization and customization. Journal of Services Marketing, 33(1), 73-87. https://doi.org/10.1108/JSM-10-2018-0285

Gürel, E. (2017). SWOT Analysis: A theoretical Review. Journal of International Social Research, 10(51), 994-1006. https://doi.org/10.17719/jisr.2017.1832 
Haab, H., Bieber, D. \& Elfert, P. (2019). Zwischen Interaktionsarbeit und ServiceEngineering - Auf dem Weg zu einem integrativen Ansatz in der Dienstleistungsforschung. In V. Stich, J. H. Schumann, D. Beverungen, G. Gudergan \& P. Jussen (Hrsg.), Digitale Dienstleistungsinnovationen (Bd. 10, S. 49-71). Springer Berlin Heidelberg. https://doi.org/10.1007/978-3-662-59517-6_3

Hacker, W. (2009). Arbeitsgegenstand Mensch: Psychologie dialogisch-interaktiver Erwerbsarbeit: Ein Lehrbuch. Pabst Science Publishers.

Hacker, W. (2018a). Arbeitsgestaltung bei dialogisch-interaktiver Er-werbsarbeit. In ver.di-Bereich Innovation und Gute Arbeit (Hrsg.), Arbeiten mit Menschen - Interaktionsarbeit humanisieren. Band 1: Gestaltungskonzepte und Forschungsbedarf (S. 31-35). Bund-Verlag GmbH.

Hacker, W. (2018b). Menschengerechtes Arbeiten in der digitalisierten Welt: Eine Wissenschaftliche Handreichung (1. Aufl.). Mensch - Technik - Organisation: Band 49. vdf Hochschulverlag AG an der ETH Zürich.

Hacker, W. \& Richter, P. (1980). Psychologische Bewertung von Arbeitsgestaltungsmaßnahmen: Ziele und Bewertungsmaßstäbe. Spezielle Arbeits- und Ingenieurpsychologie in Einzeldarstellungen. Springer.

Hielscher, V., Nock, L., Kirchen-Peters, S. \& Blass, K. (2013). Zwischen Kosten, Zeit und Anspruch: Das alltägliche Dilemma sozialer Dienstleistungsarbeit. Springer Fachmedien Wiesbaden. https://doi.org/10.1007/978-3-658-01378-3

Hülsken-Giesler, M. (2015). Technik und Neue Technologien in der Pflege. In H. Brandenburg \& S. Dorschner (Hrsg.), Verlag Huber Programmbereich Pflege. Pflegewissenschaft: Lehr- und Arbeitsbuch zur Einführung in die Pflegewissenschaft (S. 262-280). Huber.

Hülsken-Giesler, M. \& Daxberger, S. (2018). Robotik in der Pflege aus pflegewissenschaftlicher Perspektive. In O. Bendel (Hrsg.), Pflegeroboter (S. 125-139). Springer Fachmedien Wiesbaden. https://doi.org/10.1007/978-3-65822698-5_7

Institut DGB-Index Gute Arbeit (Hrsg.). (2018). DGB-Index Gute Arbeit Der Report 2018: Wie die Beschäftigten die Arbeitsbedingungen in Deutschland beurteilen. 
Jaakkola, E. (2011). Unraveling the practices of "productization" in professional service firms. Scandinavian Journal of Management, 27(2), 221-230. https://doi.org/10.1016/j.scaman.2011.03.001

Kosow, H. \& León, C. D. (2015). Die Szenariotechnik als Methode der Expertenund Stakeholdereinbindung. In M. Niederberger \& S. Wassermann (Hrsg.), Methoden der Experten- und Stakeholdereinbindung in der sozialwissenschaftlichen Forschung (S. 217-242). Springer Fachmedien Wiesbaden. https://doi.org/10.1007/978-3-658-01687-6_11

Kristiansen, P. \& Rasmussen, R. (2014). Building a Better Business Using the Lego Serious Play Method. Wiley.

Kuhlmann, M. (2009). Beobachtungsinterview. In S. Kühl, P. Strodtholz \& A. Taffertshofer (Hrsg.), Handbuch Methoden der Organisationsforschung (S. 78-99). VS Verlag für Sozialwissenschaften. https://doi.org/10.1007/978-3-53191570-8_5

Kusay-Merkle, U. (2018). Der rote Faden: Von der Gestaltung eines KanbanBoards bis zur praktischen Arbeit mit dem Board. In U. Kusay-Merkle (Hrsg.), Agiles Projektmanagement im Berufsalltag (S. 205-210). Springer Berlin Heidelberg. https://doi.org/10.1007/978-3-662-56800-2_17

Leffingwell, D. (2011). Agile software requirements: Lean requirements practices for teams, programs, and the enterprise. The Agile software development series. Addison-Wesley.

Leimeister, J. M. (2020). Dienstleistungsengineering und -management (2. Aufl.). Springer Berlin Heidelberg. https://doi.org/10.1007/978-3-662-59858-0

Luczak, H, Volpert, W., Raeithel, A. \& Schwier, W. (1987). Arbeitswissenschaft: Kerndefinition, Gegenstandskatalog, Forschungsgebiete: Bericht an den Vorstand der Gesellschaft für Arbeitswissenschaft und die Stiftung Volkswagenwerk. RKW; TÜV Rheinland.

Mayring, P. (2015). Qualitative Inhaltsanalyse: Grundlagen und Techniken (12., überarbeitete Auflage). Beltz Verlag. http://d-nb.info/1063369835/04 
Menschner, P., Prinz, A. \& Leimeister, J. M. (2014). Service Engineering Plus Systematische Gestaltung personenbezogener Dienstleistungen. In D. Bieber \& M. Geiger (Hrsg.), Personenbezogene Dienstleistungen im Kontext komplexer Wertschöpfung: Anwendungsfeld "Seltene Krankheiten" (S. 203-211). Springer Fachmedien Wiesbaden.

Meyer, K. \& Zinke, C. (2018). Service Engineering - eine Standortbestimmung. In K. Meyer, S. Klingner \& C. Zinke (Hrsg.), Service Engineering (Bd. 30, S. 3-17). Springer Fachmedien Wiesbaden. https://doi.org/10.1007/978-3-65820905-6_1

Müller, N. \& Thorein, A. (2017). Arbeit mit Menschen humanisieren. Gute Arbeit, 9, 8-12.

Murray, A. \& Scuotto, V. (2015). The Business Model Canvas: A tool for Market Driven Entrepreneurs. Symphonya. Emerging Issues in Management, 3, 94-109. https://doi.org/10.4468/2015.3.13murray.scuotto

Pangert, B., Pauls, N. \& Schüpbach, H. (2016). Die Auswirkungen arbeitsbezogener erweiterter Erreichbarkeit auf Life-Domain-Balance und Gesundheit: Forschung Projekt F 2353. Dortmund, Berlin, Dresden. BAuA.

Pietzcker, F. \& Looks, P. (Hrsg.). (2010). Mensch - Technik - Organisation: Bd. 45. Der Aufgabenbezogene Informationsaustausch: Zeitweilige partizipative Gruppenarbeit zur Problemlösung. Mit besonderem Blick auf Organisationsentwicklung, Wissensmanagement und betriebliche Gesundheitsvorsorge. vdf Hochschulverlag.

Kyhnau, J., \& Nielsen, C. (2015). Review of Value Proposition Design: How to create products and services customers want. Journal of Business Models, 3(1), 81-89. https://doi.org/10.5278/ojs.jbm.v3i1.1105

Rieder, K. (2018). Humane Gestaltung für die Arbeit von Kundinnen und Kunden. In ver.di-Bereich Innovation und Gute Arbeit (Hrsg.), Arbeiten mit Menschen - Interaktionsarbeit humanisieren. Band 1: Gestaltungskonzepte und Forschungsbedarf (S. 77-79). Bund-Verlag GmbH.

Rieder, K. \& Voß, G. G. (2009). Der Arbeitende Kunde. Entwicklung eines neuen Typus des Konsumenten. Wirtschaftspsychologie, 1, 4-10. 
Robra-Bissantz, S., Lux, A. \& Lattemann C. (2021). Service for Good: Grundlagen und Methoden zum Design personennaher Dienstleistungen. InformatikSpektrum, 44(4), (im Erscheinen).

Schat, H.-D. (2005). Ideen fürs Ideenmanagement: Betriebliches Vorschlagswesen (BVW) und Kontinuierlichen Verbesserungsprozess (KVP) gemeinsam realisieren. Wirtschaftsverlag Bachem.

Schlick, C., Bruder, R., Luczak, H. (2018). Arbeitswissenschaft (4. Auflage). Springer.

Schultetus, W. (2006). Arbeitswissenschaft - von der Theorie zur Praxis: Arbeitswissenschaftliche Erkenntnisse und ihr wirtschaftlicher Nutzen. Wirtschaftsverlag Bachem.

Shire, K. A. \& Leimeister, J. M. (Hrsg.). (2012). Springer-Gabler Research. Technologiegestützte Dienstleistungsinnovation in der Gesundheitswirtschaft. Springer-Gabler.

Slesina, W. \& Broekmann, M. (1992). Gesundheitszirkel zur Verstärkung des Gesundheitsschutzes im Betrieb. Arbeit, 1(2). https://doi.org/10.1515/arbeit1992-0204

Smith, M. L. \& Erwin, J. (2005). Role \& responsibility charting (RACI). Project Management Forum (PMForum).

Teixeira, J., Patrício, L., Nunes, N. J., Nóbrega, L., Fisk, R. P. \& Constantine, L. (2012). Customer experience modeling: from customer experience to service design. Journal of Service Management, 23(3), 362-376. https://doi.org/10.1108/09564231211248453

Thorein, A. \& Riesenberg-Mordeja, H. (2018). Gute (Dienstleistungs-)Arbeit der Zukunft: Anforderungen von ver.di an Prävention 4.0. In O. Cernavin, W. Schröter \& S. Stowasser (Hrsg.), Prävention 4.0 (S. 109-120). Springer Fachmedien Wiesbaden. https://doi.org/10.1007/978-3-658-17964-9_7

Tuckman, B. W. \& Jensen, M. A. C. (1977). Stages of Small-Group Development Revisited. Group \& Organization Studies, 2(4), 419-427. https://doi.org/10.1177/105960117700200404

Ulich, E. (2005). Arbeitspsychologie (6. Aufl.). vdf Hochschulverlag AG an der ETH Zürich; Schöffer-Poeschel Verlag. 
Ulich, E., Zink, K. J. \& Kubek, V. (2013). Das Menschenbild in Arbeitswissenschaft und Betriebswirtschaftslehre. Zeitschrift für Arbeitswissenschaft, 67(1), 15-22. https://doi.org/10.1007/BF03373899

van Husen, C., Abdel Razek, A. R. \& Hengels, A. (2020). Service Prototyping umsetzen. In C. van Husen \& J. Ovtcharova (Hrsg.), Multidimensionales Service Prototyping (S.131-171). Springer Berlin Heidelberg. https://doi.org/10.1007/978-3-662-60732-9_3

Wendsche, J. \& Lohmann-Haislah, A. (2018). Arbeitspausen gesundheits- und leistungsförderlich gestalten (1. Aufl.). Managementpsychologie: Band 3. Hogrefe.

Werbach, K. \& Hunter, D. (2012). For the Win: How Game Thinking Can Revolutionize Your Business. Wharton Digital Press.

Widuckel, W. (2015). Arbeitskultur 2020 - Herausforderungen für die Zukunft der Arbeit. In W. Widuckel, K. de Molina, M. J. Arbeitskultur 2020 (S. 27-44). Springer Gabler. https://doi.org/10.1007/978-3-658-06092-3_2 


\section{Impressum}

Die vorliegende Publikation ist ein Ergebnis des Forschungsprojektes SO-SERVE (Social Service Engineering - Synergien von Arbeits- und Dienstleistungswissenschaft für die Verbesserung von Arbeit an und mit Menschen nutzen).

Das Forschungs- und Entwicklungsprojekt SO-SERVE wird im Rahmen des Programms "Innovationen für die Produktion, Dienstleistung und Arbeit von morgen" (Förderkennzeichen 02L18A180 ff.) vom Bundesministerium für Bildung und Forschung (BMBF) und dem Europäischen Sozialfonds (ESF) gefördert und vom Projektträger Karlsruhe (PTKA) betreut. Die Verantwortung für den Inhalt dieser Veröffentlichung liegt bei den Autoren.“

Ziel der Europäischen Union ist es, dass alle Menschen eine berufliche Perspektive erhalten. Der Europäische Sozialfonds (ESF) verbessert die Beschäftigungschancen, unterstützt die Menschen durch Ausbildung und Qualifizierung und trägt zum Abbau von Benachteiligungen auf dem Arbeitsmarkt bei.

Mehr zum ESF unter: www.esf.de.

Herausgebende Institutionen

TU Dresden

Bereich Mathematik und Naturwissenschaften, Fakultät Psychologie Arbeitsgruppe Wissen-DenkenHandeln

01062 Dresden

Telefon +49 351 463-36890

agwdh@tu-dresden.de
Universität Leipzig

Universitätsrechenzentrum

Abteilung Forschung und Entwicklung

Neues Augusteum

Augustusplatz 10

04109 Leipzig

Telefon +49 341 97-33303 


\section{Zitierhinweis}

Friedrich, J.; Gilbert, G.; Pietrzyk, U.; Römer, V.; Steputat-Rätze, A.; Zinke-Wehlmann, C. (2021): Perspektiven auf die Gestaltung von Interaktionsarbeit. Whitepaper. https://doi.org/10.25368/2021.4

Bildnachweise

Titelbild: Tobias Gilbert

Seite 6, 13, 23, 27, 39, 43: Plxoboy = 
ISBN: 978-3-86780-678-7 